\title{
STREAM II-V4: Revision for STREAM II-V3 to Allow Mouse-Driven Selection of Release Location from a Graphical User Interface (U)
}

Kuo-Fu Chen

Gary L. Snyder

Savannah River Technology Center

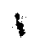

Publication Date: October 9, 2002

DOES NOT CONTAIN

UNCLASSIFIED CONTROLLED

NUCLEAR INFORMATION

ADC \&

Reviewing
Official:

Date: $10 \cdot 25-02$

Westinghouse Savannah River Company

Savannah River Site

Aiken, SC 29808

This document was prepared in connection with work done under Contract No. DE-AC09-96SR18500

with the U.S. Department of Energy 
This document was prepared in conjunction with work accomplished under Contract No. DE-AC09-96SR18500 with the U. S. Department of Energy.

\section{DISCLAIMER}

This report was prepared as an account of work sponsored by an agency of the United States Government. Neither the United States Government nor any agency thereof, nor any of their employees, makes any warranty, express or implied, or assumes any legal liability or responsibility for the accuracy, completeness, or usefulness of any information, apparatus, product or process disclosed, or represents that its use would not infringe privately owned rights. Reference herein to any specific commercial product, process or service by trade name, trademark, manufacturer, or otherwise does not necessarily constitute or imply its endorsement, recommendation, or favoring by the United States Government or any agency thereof. The views and opinions of authors expressed herein do not necessarily state or reflect those of the United States Government or any agency thereof.

This report has been reproduced directly from the best available copy.

Available for sale to the public, in paper, from: U.S. Department of Commerce, National Technical Information Service, 5285 Port Royal Road, Springfield, VA 22161, phone: (800) 553-6847, fax: (703) 605-6900

email: orders@ntis.fedworld.gov

online ordering: http://www.ntis.gov/help/index.asp

Available electronically at http://www.osti.gov/bridge

Available for a processing fee to U.S. Department of Energy and its contractors, in paper, from: U.S. Department of Energy, Office of Scientific and Technical Information, P.O. Box 62, Oak Ridge, TN 37831-0062,

phone: (865)576-8401,

fax: (865)576-5728

email: $\underline{\text { reports@ adonis.osti.gov }}$ 
WSRC-TR-2002-00470

October 2002

DOCUMENT: $\quad$ WSRC-TR-2002-00470

TITLE: STREAM II-V4: Revision for STREAM II-V3 to Allow Mouse-Driven Selection of Release Location from a Graphical User Interface (U)

TASK:

TECHNICAL REVIEW

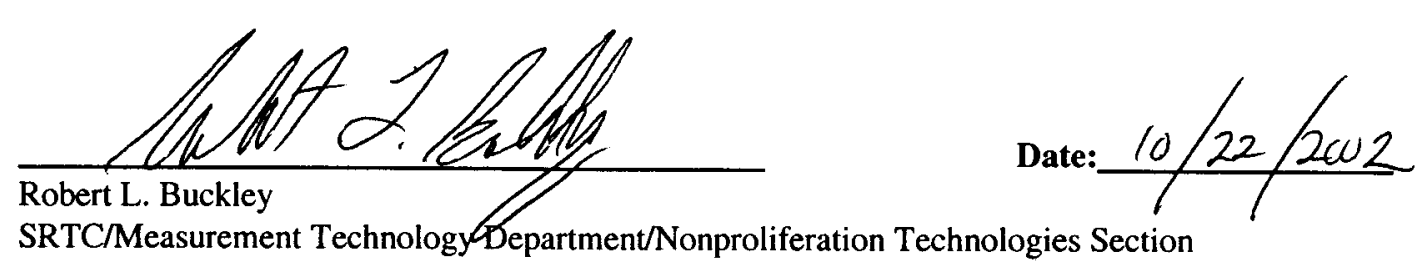

APPROVALS

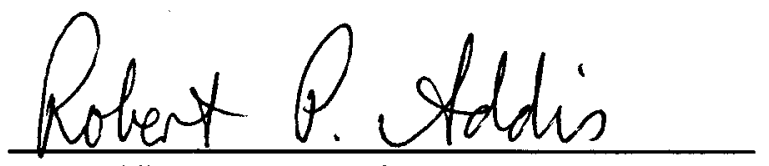

Date: $10 / 22 / 2002$

R. P. Addie, Manager, ATG

SRTC/Measurement Technology Department/Nonproliferation Technologies Section

ACAri

Date: $10-25=02$

A. L. Bone, Manager, NTS

SRTC/Measurement Technology Department/Nonproliferation Technologies Section

iii 
WSRC-TR-2002-00470

October 2002

(Blank Page) 


\section{ABSTRACT}

STREAM II-V3 is an aqueous transport module of the Savannah River Site emergency response Weather INformation Display (WIND) system. STREAM II-V3 predicts peak concentration and peak concentration arrival time at downstream locations for releases from the SRS facilities to the Savannah River. Fifteen pre-determined potential release locations from SRS facilities were built into the current STREAM II-V3 model. Therefore, STREAM II-V3 can not be used for situations in which release locations differ from the fifteen pre-determined locations. To eliminate this limitation, STREAM II-V3 was upgraded. The revised STREAM II-V4 allows users to select the release location anywhere along the specified SRS main streams or the Savannah River by mouse clicking on a map displayed on the computer monitor. 


\section{TABLE OF CONTENTS}

1. Introduction

2. Implementation

2.1 Determination of the Stream Model Receiving the Discharge

2.2 Determination of the Computational Node Receiving the Discharge

3 Conclusion

References

Appendix 1 
WSRC-TR-2002-00470

October 2002

\section{LIST OF TABLES}

Table 1. Mapping between the Stream Models and the Shapefile Points on the Map

\section{LIST OF FIGURES}

Figure 1. STREAM II-V3 User Interface Screen for Data Input 5

Figure 2. Shapefile Points Representing the Segment Center in Red 6

$\begin{array}{lll}\text { Figure 3. Model Schematics for Savannah River (not to scale) } & 7\end{array}$

Figure 4. Model Schematics for Upper Three Runs Creek (not to scale) 8

Figure 5. Model Schematics for McQueen Branch (not to scale) 9

Figure 6. Model Schematics for Tims Branch (not to scale) 10

Figure 7. Model Schematics for Beaver Dam Creek (not to scale) 11

Figure 8. Model Schematics for Fourmile Branch (not to scale) 12

Figure 9. Model Schematics for Pen Branch (not to scale) 13

Figure 10. Model Schematics for Steel Creek (not to scale) 14

Figure 11. Model Schematics for Lower Three Runs Creek (not to scale) 15 
WSRC-TR-2002-00470

October 2002

(Blank Page) 


\section{Introduction}

STREAM II-V3 [1] is an aqueous transport module of the Savannah River Site emergency response Weather INformation Display (WIND) system [2]. STREAM II-V3 predicts peak concentration and peak concentration arrival time at downstream locations for releases from the SRS facilities to the Savannah River. Fifteen pre-determined potential release locations from SRS facilities were built into the current STREAM II-V3 model, as shown in Figure 1. Therefore, STREAM II-V3 can not be used for situations in which release locations differ from the fifteen pre-determined locations. To eliminate this limitation, STREAM II-V3 was upgraded. The revised STREAM II-V4 allows users to select the release location anywhere along the specified SRS main streams or the Savannah River by mouse clicking on a map displayed on the computer screen.

\section{Implementation}

STREAM II solves a finite difference approximation of the transport equation for a model network that represents the important characteristics of the real water body. The model network divides the real water body into segments. Each segment represents a computational node. The idea is to develop a procedure to map the mouse selected release location to the computational model. The mapping procedure is presented next.

\subsection{Determination of the Stream Model Receiving the Discharge}

The STREAM II graphic user interface map, shown in Figure 1, is based on data from the ArcView GIS system [3]. ArcView GIS is a desktop computer based geographic information system. ArcView GIS displays the geographic features on a map and stores information about the features in a database and links the information to the features. This information is referred to as attribute information. ArcView GIS uses three basic shapes (point, line and polygon) to represent geographic features. For example, ArcView GIS represents buildings as points, streams as lines, and countries as polygons. The file structure of ArcView GIS to store the location, shape and attribute information of a geographic feature is referred to as a shapefile. Figure 2 shows the onsite main streams and part of the Savannah River. ArcView GIS was used to develop a point shapefile along the SRS main streams and the Savannah River, shown in red points in Figure 2. Each point has a unique identification number and a map coordinate (attribute information), as shown in Appendix 1. These points correspond to the center of the model segment. The distance between the points is 500 meters except the points for Fourmile Branch. The distance between the points for Fourmile Branch is 150 meter [4]. There are 928 points. Table 1 shows the mapping relationship between the stream models and the shapefile 
point IDs. For instance, point numbers 1 to 550 correspond to the Savannah River model segments. Successively higher number points correspond to other major streams within SRS.

When a user selects a release location by clicking the mouse at the map on the screen, the coordinates of that location are passed to the computer code. The distances between the mouse selected location and the shapefile points are calculated. The shapefile point that has the shortest distance (closest to the mouse selected location) is selected to receive the discharge. Then, the stream that receives the discharge, thus the stream model, is determined from Table 1. It is very important to note that this assumption is limited to the situation that the selected location is very close to the streams. If the selected location is far away from the streams, the topographic shape of the surface instead of the shortest distance will control the flow direction of the released material.

\subsection{Determination of the Computational Node Receiving the Discharge}

Figure 3 to 11 shows the computational model for the Savannah River, Upper Three Runs Creek, McQueen Branch, Tims Branch, Beaver Dam Creek, Fourmile Branch, Pen Branch, Steel Creek, and Lower Three Runs Creek, respectively. For a given shapefile point ID and stream model, the computational node that receives the discharge is determined from Table 1.

The selected stream model and the receiving node are used in the transport calculation.

\section{Conclusion}

The release locations in STREAM II-V3 are pre-determined. Therefore, STREAM II-V3 can not be used for situations in which releases occur at sites other than the pre-determined locations. To eliminate this limitation, STREAM II-V3 was upgraded to handle releases to the SRS main streams and the Savannah River for points other than the pre-determined locations. The revised STREAM II-V4 allows users to select the release locations anywhere along the specified SRS main streams and the Savannah River by mouse clicking at the map shown on the computer screen. It is assumed that the shapefile point with the shortest distance to the mouse selected location is selected to receive the discharge. Therefore, it is very important to note that this assumption is limited to the situation that the selected location is very close to the streams. If the selected location is far away from the streams, the topographic shape of the surface instead of the shortest distance will control the flow direction of the released material. 
References

1. Chen, Kuo-Fu, "STREAM II-V3: Revision for STREAM II-V2 to Include the Sedimentation Effects on a Release from H-Area (U)," WSRC-TR-2001-00539, Savannah River Site, December 1, 2001.

2. Hunter, C. H., "Weather Information and Display (WIND) System User's Manual," WSRCTM-90-14, Savannah River Site, 1990.

3. ArcView GIS Version 3.0a, Copyright (C), Environmental Systems Research Institute, Inc.

4. Chen, Kuo-Fu, "STREAM II for SRS Aqueous Release Emergency Response (U)," WSRC-TR-98-00234, Savannah River Site, June, 1998. 
Table 1 Mapping Between the Stream Models and the Shapefile Points on the MAP

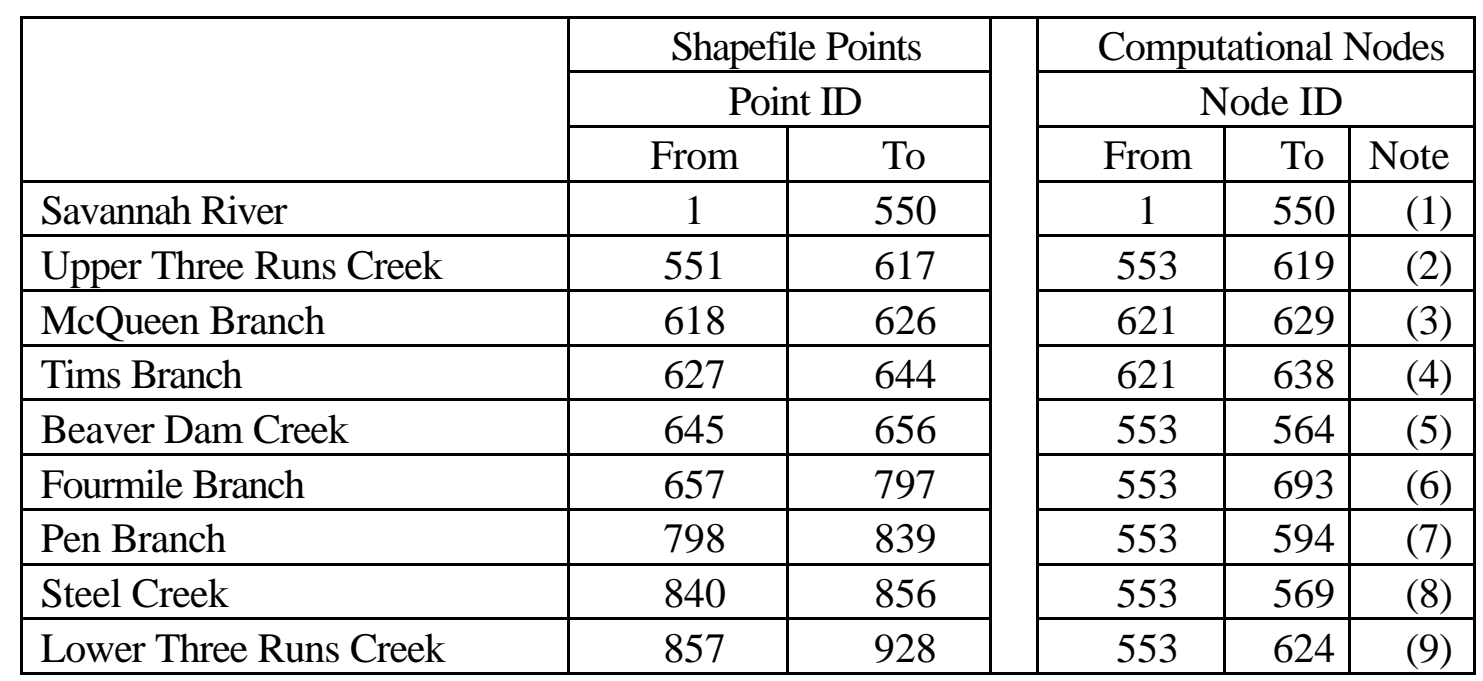
(1): Refer to Figure 3
(2): Refer to Figure 4
(3): Refer to Figure 5
(4): Refer to Figure 6
(5): Refer to Figure 7
(6): Refer to Figure 8
(7): Refer to Figure 9
(8): Refer to Figure 10
(9): Refer to Figure 11 
Figure 1 STREAM II-V3 User Interface Screen for Data Input

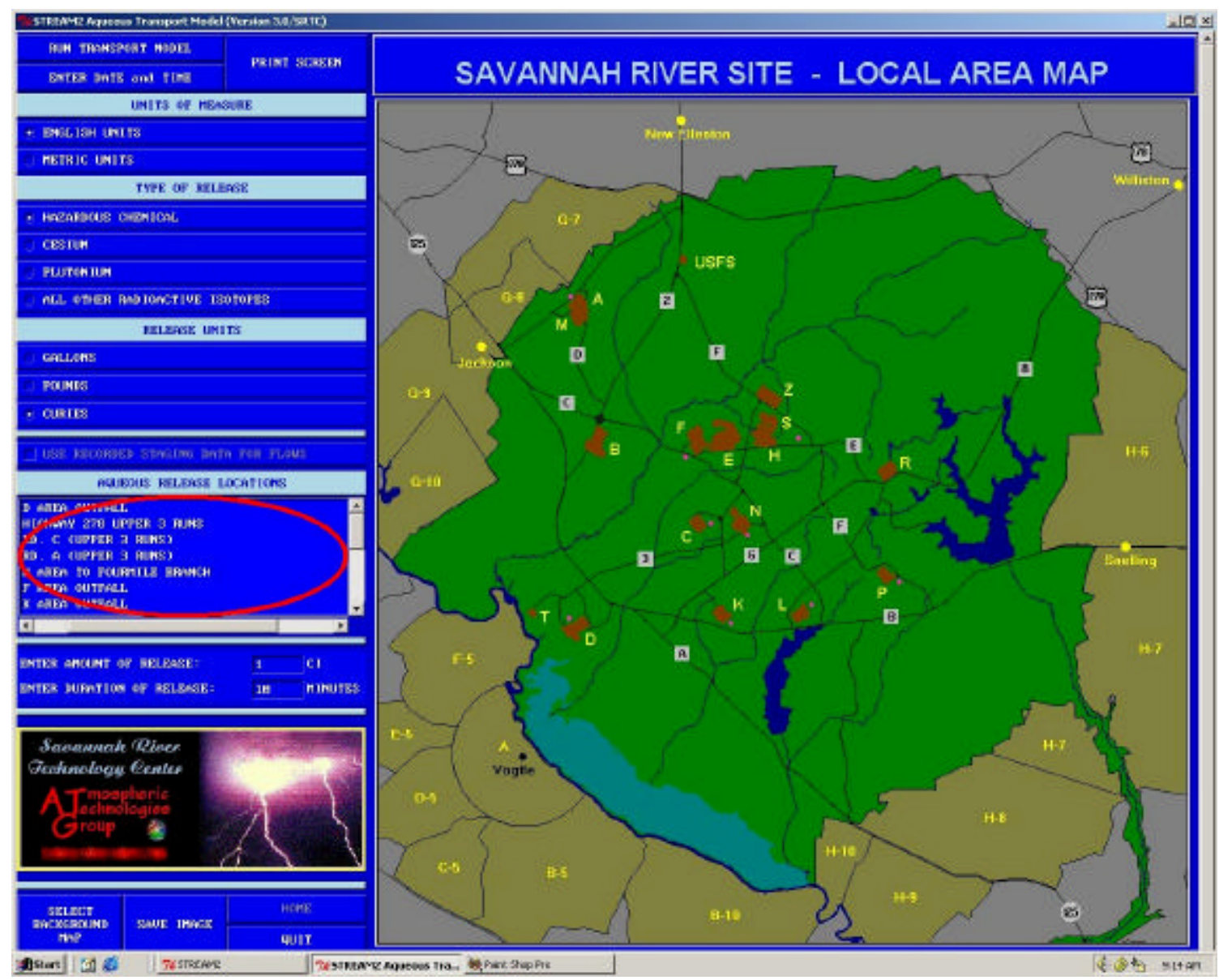

The pre-defined release locations are circled in red color. 
Figure 2 Shapefile Points Representing the Segment Center Shown in Red

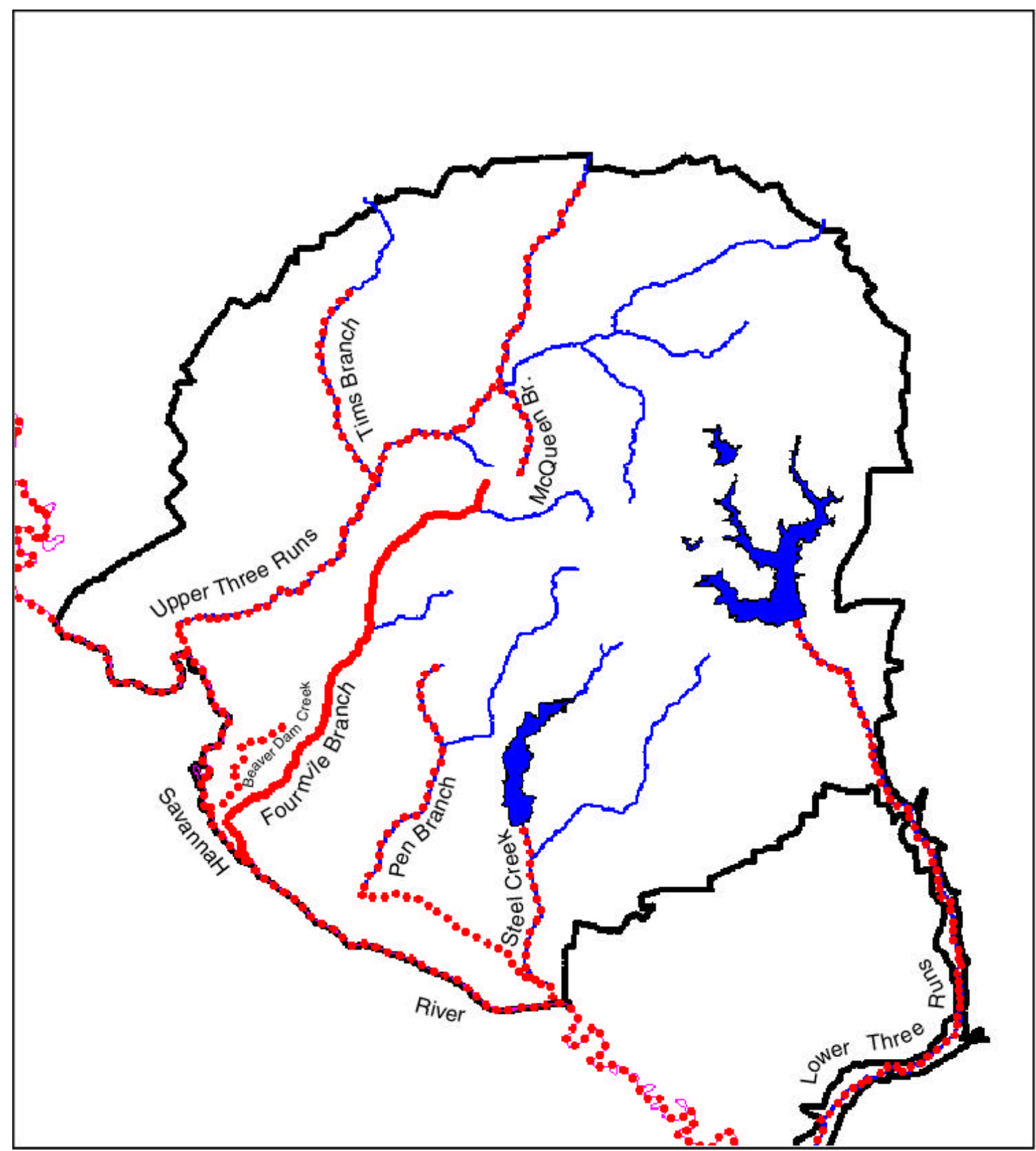

Note: The space between the points is 500 meters except the points for Fourmile Branch. The space between the points for Fourmile Branch is 150 meters. 
WSRC-TR-2002-00470

October 2002

Figure 3 
Model Schematics for Savannah River (not to scale)

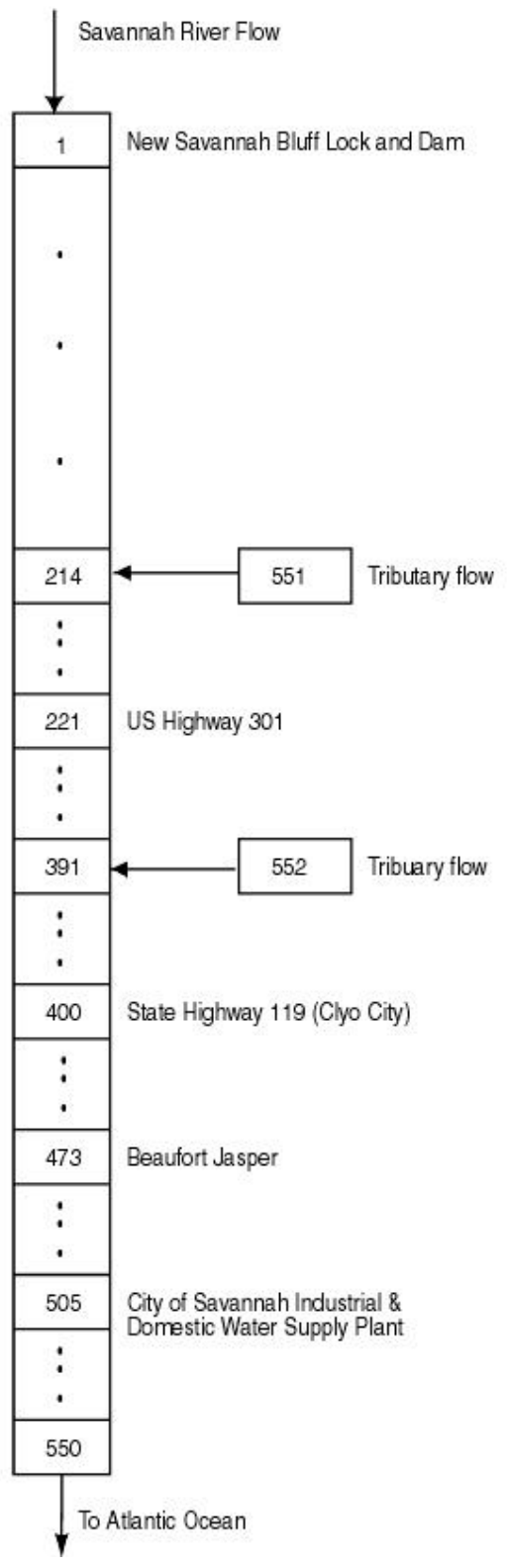


WSRC-TR-2002-00470

October 2002

Figure 4

Model Schematics for Upper Three Runs Creek (not to scale)

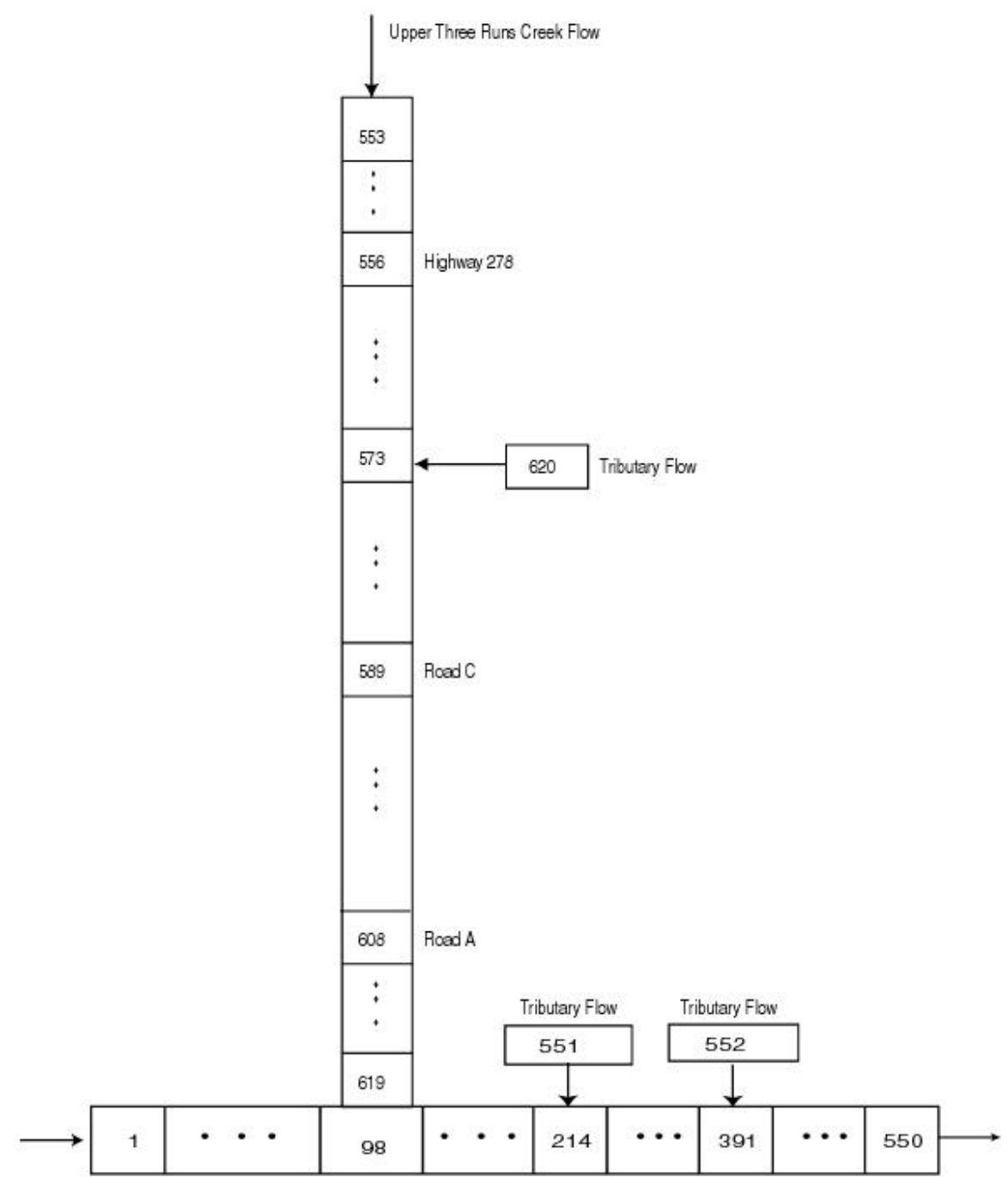

Savannah River Flow

Savannah River Flow 
Figure 5

Model Schematics for McQueen Branch (not to scale)

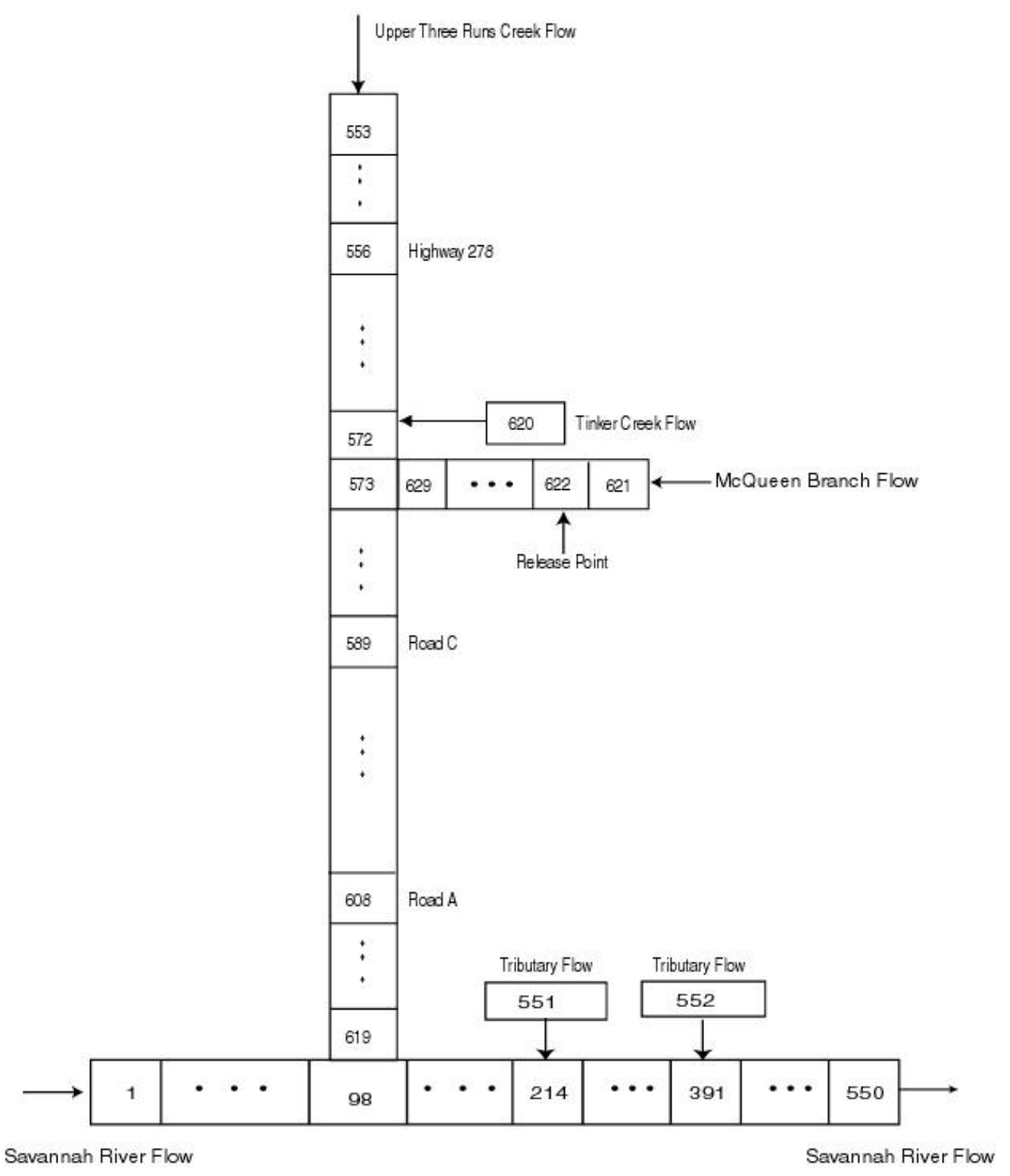


WSRC-TR-2002-00470

October 2002

Figure 6

Model Schematics for Tims Branch (not to scale)

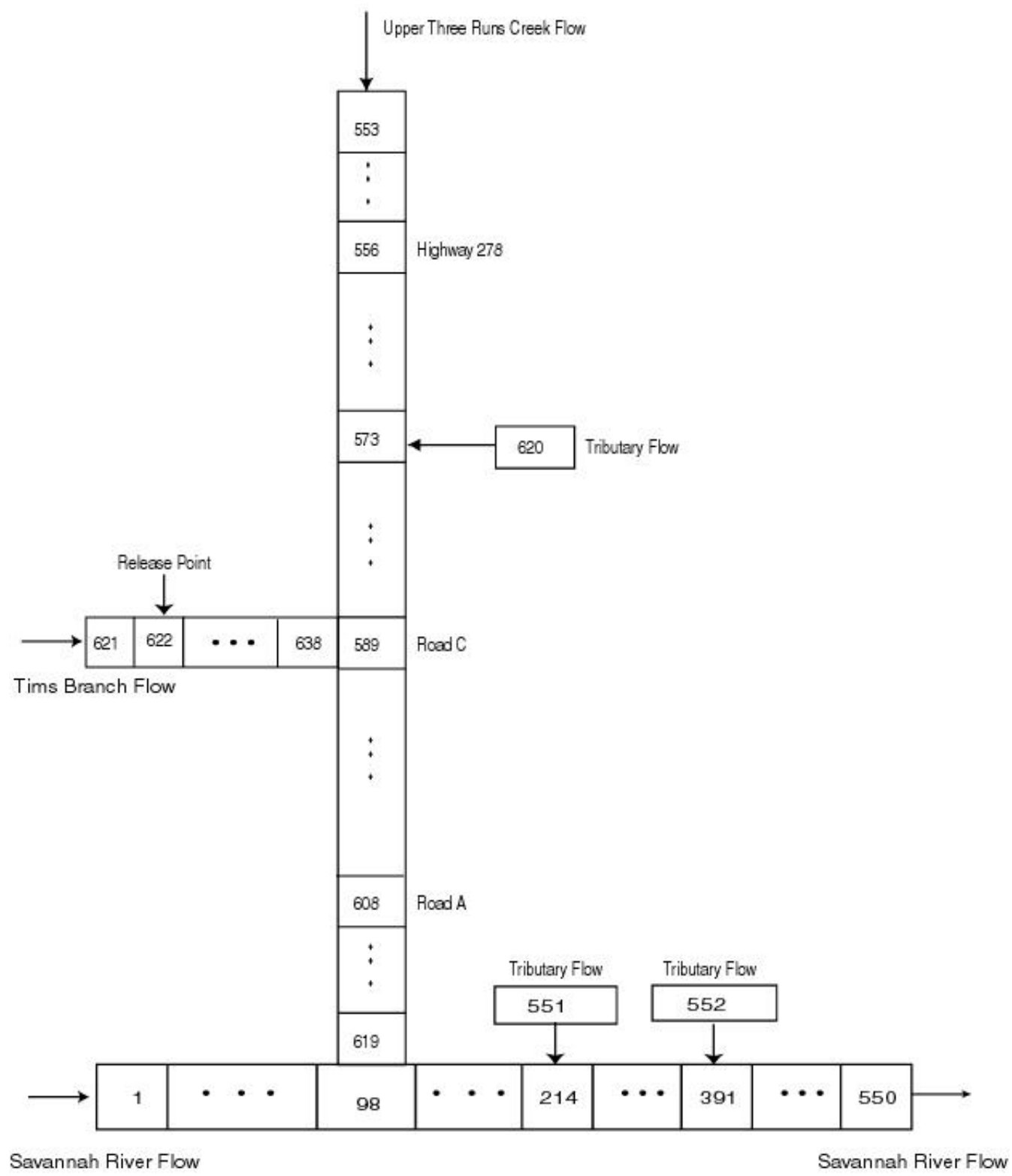


Figure 7

Model Schematics for Beaver Dam Creek (not to scale)

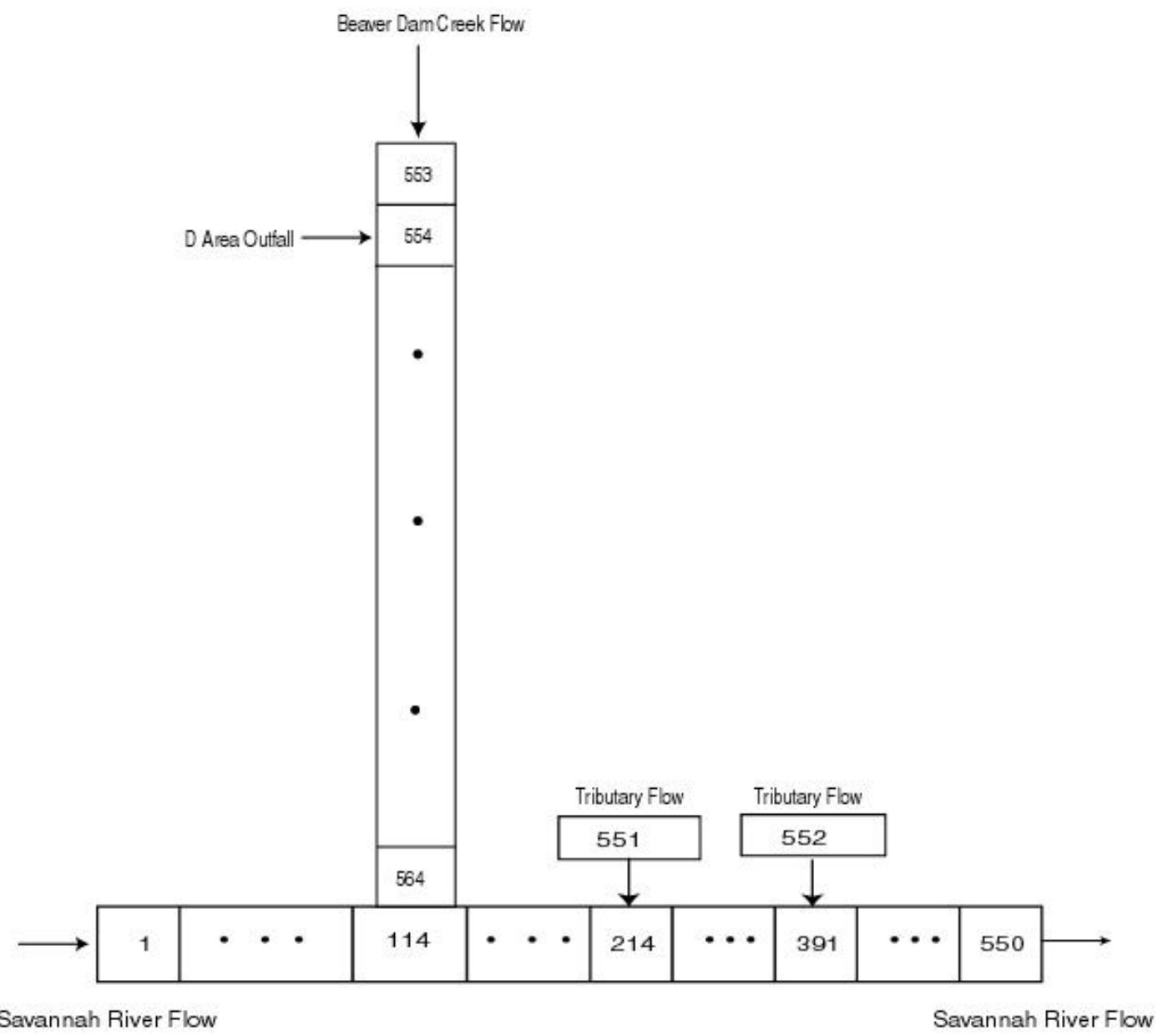


Figure 8

Model Schematics for Fourmile Branch (not to scale)

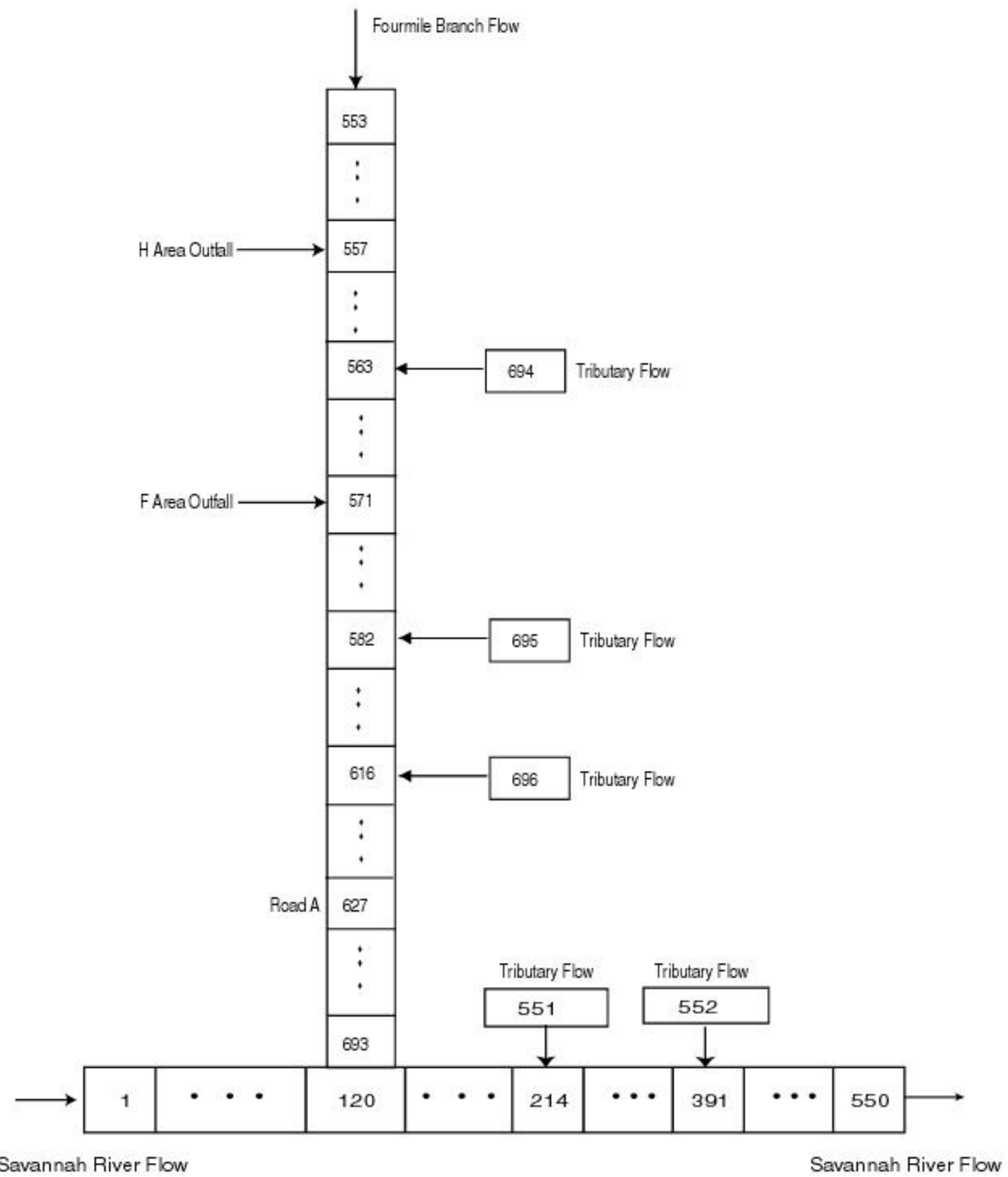


Figure 9

Model Schematics for Pen Branch (not to scale)

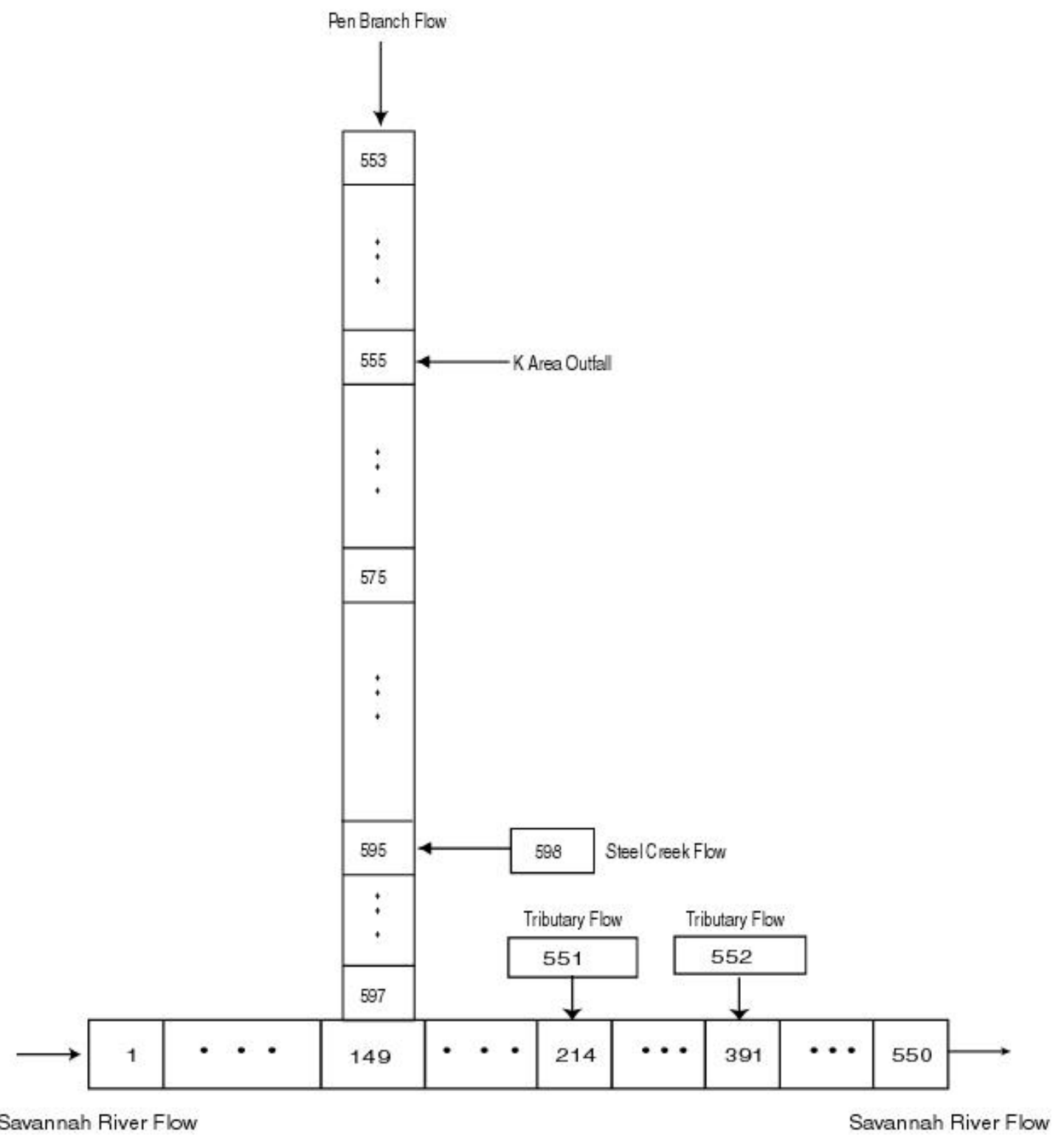


WSRC-TR-2002-00470

October 2002

Figure 10

Model Schematics for Steel Creek (not to scale)

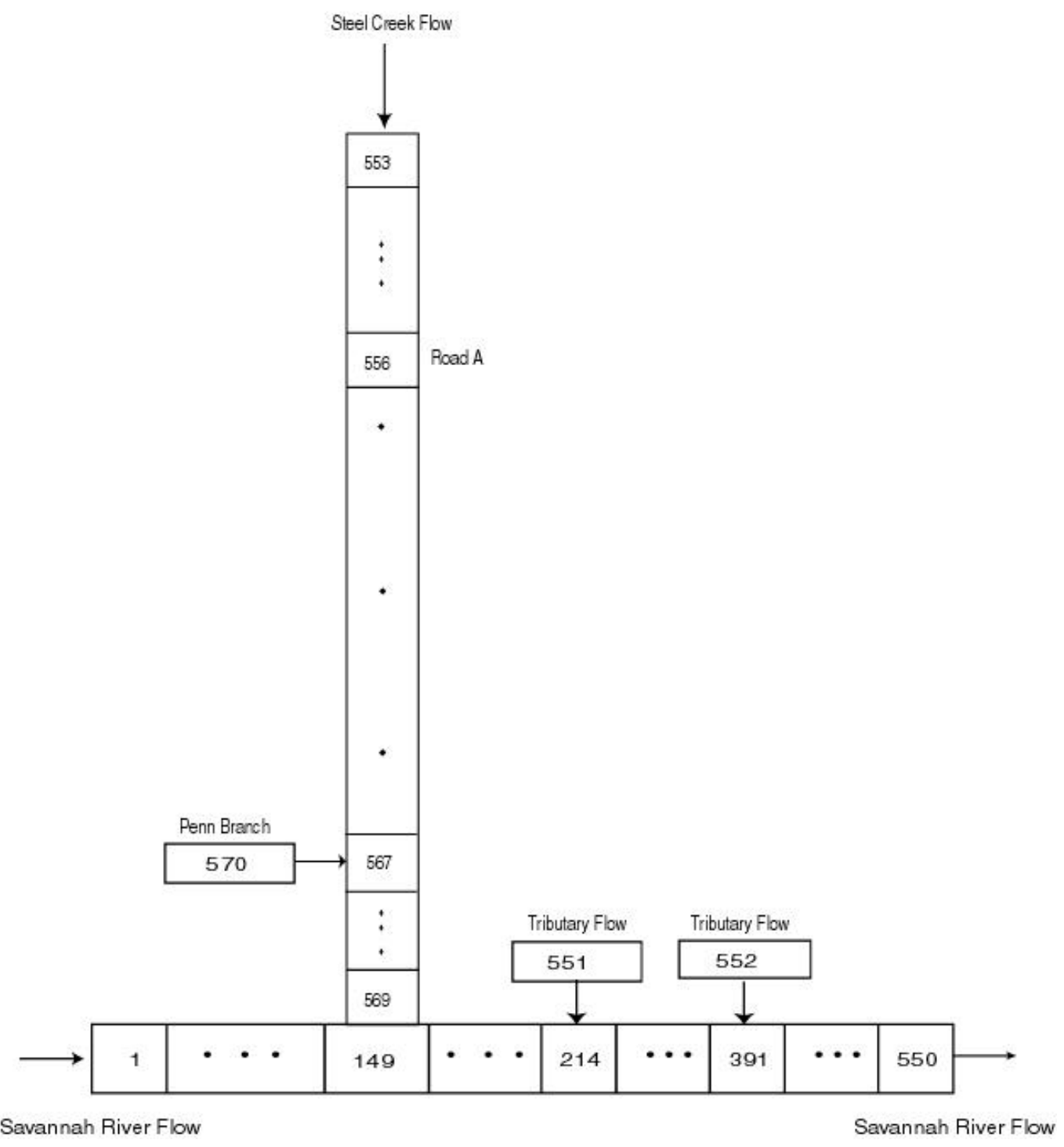


Figure 11

Model Schematics for Lower Three Runs Creek (not to scale)

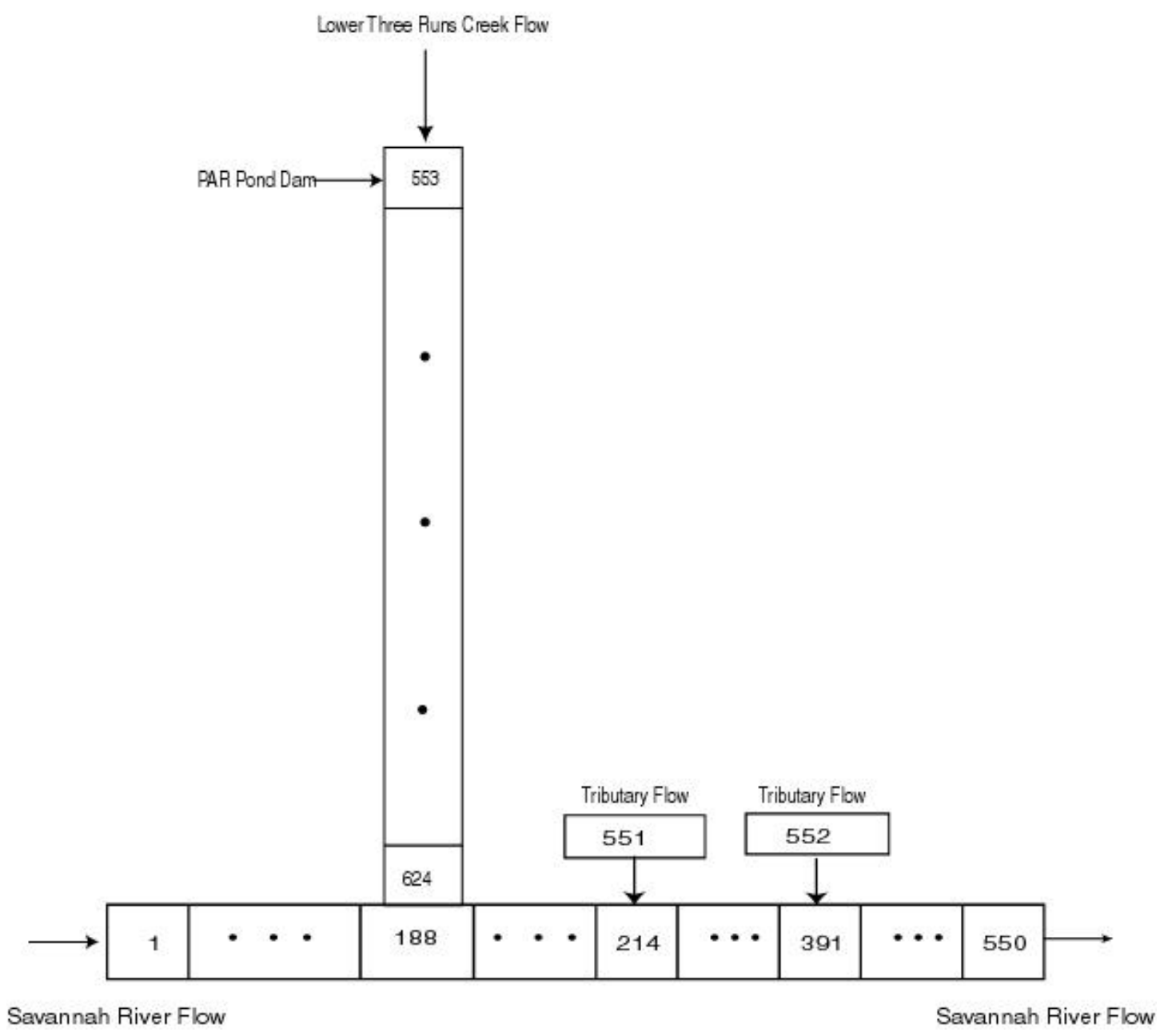


WSRC-TR-2002-00470

October 2002

Appendix 1 Shape File Points (Coordinates of the Segment Center)

\begin{tabular}{|c|c|c|c|c|}
\hline \multirow{2}{*}{$\begin{array}{l}\text { Shape File } \\
\text { Point ID }\end{array}$} & \multicolumn{2}{|c|}{ Coordinate in Lambert Projection for SRS } & \multicolumn{2}{|c|}{ Coordinate in Lambert Projection for Southeast US } \\
\hline & $\begin{array}{c}\text { Longitude } \\
\mathrm{m}\end{array}$ & $\begin{array}{l}\text { Latitude } \\
\text { m }\end{array}$ & $\begin{array}{l}\text { Longitude } \\
\text { m }\end{array}$ & $\begin{array}{l}\text { Latitude } \\
\text { m }\end{array}$ \\
\hline 1 & 112170.60 & 41685.52 & 2418221.88 & 1216791.89 \\
\hline 2 & 111867.51 & 41333.40 & 2417998.14 & 1216385.06 \\
\hline 3 & 111998.93 & 40860.43 & 2418224.21 & 1215949.68 \\
\hline 4 & 112319.51 & 40480.24 & 2418616.11 & 1215644.06 \\
\hline 5 & 112719.03 & 40184.14 & 2419067.86 & 1215436.93 \\
\hline 6 & 112984.89 & 39782.50 & 2419410.68 & 1215099.04 \\
\hline 7 & 112823.16 & 39323.66 & 2419347.20 & 1214617.00 \\
\hline 8 & 112498.30 & 38954.57 & 2419105.67 & 1214189.07 \\
\hline 9 & 112639.32 & 38531.28 & 2419330.89 & 1213804.25 \\
\hline 10 & 113128.76 & 38481.39 & 2419819.78 & 1213856.43 \\
\hline 11 & 113596.26 & 38639.74 & 2420244.26 & 1214107.71 \\
\hline 12 & 114060.65 & 38578.06 & 2420711.09 & 1214143.21 \\
\hline 13 & 114368.60 & 38184.26 & 2421093.48 & 1213821.67 \\
\hline 14 & 114484.43 & 37994.39 & 2421245.91 & 1213659.89 \\
\hline 15 & 114591.67 & 37859.57 & 2421378.60 & 1213550.19 \\
\hline 16 & 114416.04 & 37407.77 & 2421300.07 & 1213072.16 \\
\hline 17 & 114769.02 & 37085.29 & 2421711.78 & 1212829.64 \\
\hline 18 & 115201.89 & 36841.90 & 2422185.28 & 1212680.94 \\
\hline 19 & 115474.13 & 36654.47 & 2422490.18 & 1212553.84 \\
\hline 20 & 115692.49 & 36572.45 & 2422720.63 & 1212518.69 \\
\hline 21 & 115933.97 & 37002.65 & 2422868.00 & 1212989.20 \\
\hline 22 & 116110.66 & 37223.38 & 2422995.23 & 1213241.50 \\
\hline 23 & 116398.69 & 37107.68 & 2423300.77 & 1213187.80 \\
\hline 24 & 116442.33 & 36653.96 & 2423437.06 & 1212753.12 \\
\hline 25 & 116262.60 & 36236.36 & 2423347.48 & 1212307.67 \\
\hline 26 & 116677.65 & 36047.76 & 2423792.27 & 1212208.90 \\
\hline 27 & 117143.97 & 36042.05 & 2424249.45 & 1212299.53 \\
\hline 28 & 117003.88 & 35609.33 & 2424201.75 & 1211847.48 \\
\hline 29 & 116986.98 & 35268.06 & 2424255.65 & 1211510.26 \\
\hline 30 & 117422.63 & 35494.20 & 2424635.00 & 1211821.31 \\
\hline 31 & 117849.02 & 35431.47 & 2425064.91 & 1211847.96 \\
\hline 32 & 117808.58 & 34962.87 & 2425122.07 & 1211381.37 \\
\hline 33 & 117619.42 & 34537.36 & 2425024.90 & 1210926.23 \\
\hline 34 & 117854.73 & 34122.85 & 2425340.56 & 1210569.44 \\
\hline 35 & 118134.08 & 33918.42 & 2425655.93 & 1210427.19 \\
\hline 36 & 118369.25 & 34353.27 & 2425796.15 & 1210900.96 \\
\hline 37 & 118692.87 & 34722.42 & 2426036.43 & 1211328.74 \\
\hline 38 & 119003.77 & 35107.52 & 2426260.98 & 1211769.49 \\
\hline 39 & 119405.52 & 34963.95 & 2426683.47 & 1211712.02 \\
\hline 40 & 119515.07 & 34480.62 & 2426890.36 & 1211261.98 \\
\hline 41 & 119449.82 & 33996.78 & 2426926.42 & 1210775.37 \\
\hline 42 & 119763.60 & 33960.36 & 2427240.78 & 1210804.52 \\
\hline 43 & 120071.21 & 34323.46 & 2427466.64 & 1211223.09 \\
\hline 44 & 120329.49 & 34737.64 & 2427633.73 & 1211681.41 \\
\hline 45 & 120745.66 & 34517.74 & 2428086.08 & 1211552.28 \\
\hline 46 & 121132.21 & 34237.38 & 2428521.96 & 1211357.91 \\
\hline 47 & 121046.64 & 33798.94 & 2428528.78 & 1210911.49 \\
\hline 48 & 120902.77 & 33658.26 & 2428417.13 & 1210744.23 \\
\hline 49 & 120744.38 & 33532.82 & 2428288.14 & 1210588.87 \\
\hline 50 & 120611.71 & 33494.01 & 2428166.41 & 1210523.53 \\
\hline 51 & 120138.71 & 33542.56 & 2427693.84 & 1210473.37 \\
\hline 52 & 119723.11 & 33338.08 & 2427329.62 & 1210187.63 \\
\hline
\end{tabular}




\begin{tabular}{|c|c|c|c|c|}
\hline 53 & 119717.88 & 32851.44 & 2427424.95 & 1209710.66 \\
\hline 54 & 119566.85 & 32381.21 & 2427374.32 & 1209219.64 \\
\hline 55 & 119856.45 & 32001.13 & 2427735.97 & 1208907.73 \\
\hline 56 & 120326.42 & 31838.81 & 2428229.07 & 1208846.00 \\
\hline 57 & 120819.14 & 31762.74 & 2428726.62 & 1208873.31 \\
\hline 58 & 121257.03 & 31758.21 & 2429155.78 & 1208959.27 \\
\hline 59 & 121335.27 & 31292.41 & 2429328.44 & 1208519.90 \\
\hline 60 & 121623.81 & 30896.59 & 2429692.33 & 1208192.38 \\
\hline 61 & 121846.61 & 30664.14 & 2429958.19 & 1208011.06 \\
\hline 62 & 122037.28 & 30577.91 & 2430162.46 & 1207966.09 \\
\hline 63 & 122153.91 & 30429.19 & 2430307.21 & 1207844.73 \\
\hline 64 & 122161.60 & 29937.38 & 2430416.27 & 1207365.35 \\
\hline 65 & 122089.19 & 29616.86 & 2430411.63 & 1207036.96 \\
\hline 66 & 121961.55 & 29508.53 & 2430309.16 & 1206904.67 \\
\hline 67 & 121784.63 & 29483.22 & 2430141.37 & 1206843.39 \\
\hline 68 & 121504.94 & 29822.22 & 2429797.86 & 1207117.17 \\
\hline 69 & 121050.42 & 29862.01 & 2429345.16 & 1207062.26 \\
\hline 70 & 121010.36 & 29394.64 & 2429402.45 & 1206596.92 \\
\hline 71 & 121157.70 & 28929.21 & 2429642.63 & 1206172.18 \\
\hline 72 & 121624.17 & 28795.58 & 2430126.41 & 1206137.78 \\
\hline 73 & 121540.06 & 28468.92 & 2430111.58 & 1205800.96 \\
\hline 74 & 121073.78 & 28319.50 & 2429686.42 & 1205558.57 \\
\hline 75 & 120607.36 & 28274.78 & 2429239.52 & 1205418.55 \\
\hline 76 & 120537.60 & 27849.43 & 2429259.09 & 1204988.17 \\
\hline 77 & 120873.87 & 27511.27 & 2429657.76 & 1204726.88 \\
\hline 78 & 121128.02 & 27088.14 & 2429993.66 & 1204365.53 \\
\hline 79 & 121572.61 & 26869.50 & 2430473.60 & 1204243.48 \\
\hline 80 & 122002.17 & 26629.28 & 2430943.30 & 1204097.23 \\
\hline 81 & 122355.05 & 26284.59 & 2431359.57 & 1203832.99 \\
\hline 82 & 122665.59 & 25900.63 & 2431742.56 & 1203521.59 \\
\hline 83 & 123018.25 & 25601.35 & 2432149.25 & 1203301.70 \\
\hline 84 & 123491.62 & 25461.96 & 2432640.98 & 1203263.12 \\
\hline 85 & 123928.83 & 25228.99 & 2433116.68 & 1203125.55 \\
\hline 86 & 124397.70 & 25066.50 & 2433608.78 & 1203063.45 \\
\hline 87 & 124632.39 & 24651.60 & 2433923.99 & 1202706.13 \\
\hline 88 & 124640.76 & 24159.90 & 2434033.70 & 1202226.98 \\
\hline 89 & 124871.03 & 23756.64 & 2434342.18 & 1201880.13 \\
\hline 90 & 125356.67 & 23754.01 & 2434817.69 & 1201977.84 \\
\hline 91 & 125825.91 & 23632.83 & 2435301.63 & 1201956.23 \\
\hline 92 & 126267.63 & 23401.68 & 2435781.37 & 1201821.38 \\
\hline 93 & 126757.88 & 23348.90 & 2436271.75 & 1201871.01 \\
\hline 94 & 127244.05 & 23482.60 & 2436719.62 & 1202102.18 \\
\hline 95 & 127524.83 & 23857.07 & 2436916.89 & 1202526.40 \\
\hline 96 & 127398.33 & 24327.37 & 2436696.04 & 1202960.23 \\
\hline 97 & 127210.71 & 24741.02 & 2436427.12 & 1203326.03 \\
\hline 98 & 127665.62 & 24831.13 & 2436853.41 & 1203508.11 \\
\hline 99 & 128046.02 & 24547.33 & 2437284.05 & 1203309.12 \\
\hline 100 & 128397.60 & 24214.67 & 2437696.61 & 1203056.39 \\
\hline 101 & 128510.42 & 23740.87 & 2437904.80 & 1202616.31 \\
\hline 102 & 128695.04 & 23280.05 & 2438180.54 & 1202203.76 \\
\hline 103 & 128811.63 & 22796.82 & 2438394.38 & 1201755.23 \\
\hline 104 & 129197.12 & 22496.85 & 2438833.36 & 1201541.49 \\
\hline 105 & 129355.67 & 22088.92 & 2439072.68 & 1201175.27 \\
\hline 106 & 129118.11 & 21667.97 & 2438927.30 & 1200714.50 \\
\hline 107 & 129003.10 & 21191.10 & 2438913.31 & 1200224.34 \\
\hline 108 & 128533.49 & 21057.88 & 2438481.53 & 1199997.05 \\
\hline 109 & 128395.61 & 20594.85 & 2438442.32 & 1199515.70 \\
\hline 110 & 128638.01 & 20175.59 & 2438766.00 & 1199155.72 \\
\hline
\end{tabular}


WSRC-TR-2002-00470

October 2002

\begin{tabular}{|c|c|c|c|c|}
\hline 111 & 128495.02 & 19885.20 & 2438686.13 & 1198842.17 \\
\hline 112 & 128274.27 & 19622.84 & 2438524.41 & 1198539.97 \\
\hline 113 & 128460.08 & 19169.78 & 2438799.73 & 1198135.23 \\
\hline 114 & 128424.66 & 18753.80 & 2438851.01 & 1197721.05 \\
\hline 115 & 128727.94 & 18406.01 & 2439219.47 & 1197443.53 \\
\hline 116 & 128800.31 & 17915.85 & 2439391.51 & 1196979.06 \\
\hline 117 & 129142.70 & 17555.46 & 2439800.84 & 1196697.30 \\
\hline 118 & 129445.56 & 17160.83 & 2440178.58 & 1196373.88 \\
\hline 119 & 129757.72 & 16775.95 & 2440563.41 & 1196061.92 \\
\hline 120 & 130167.43 & 16517.68 & 2441017.50 & 1195893.94 \\
\hline 121 & 130444.06 & 16151.28 & 2441363.76 & 1195592.72 \\
\hline 122 & 130777.97 & 15812.27 & 2441760.40 & 1195330.12 \\
\hline 123 & 131196.62 & 15546.97 & 2442224.70 & 1195157.12 \\
\hline 124 & 131591.30 & 15254.28 & 2442671.20 & 1194952.37 \\
\hline 125 & 132000.40 & 14971.16 & 2443129.85 & 1194759.98 \\
\hline 126 & 132352.42 & 14617.46 & 2443547.24 & 1194486.75 \\
\hline 127 & 132658.08 & 14222.61 & 2443927.80 & 1194163.71 \\
\hline 128 & 133080.40 & 13961.99 & 2444394.73 & 1193996.05 \\
\hline 129 & 133533.20 & 13760.89 & 2444879.17 & 1193892.91 \\
\hline 130 & 133901.36 & 13428.93 & 2445307.88 & 1193644.29 \\
\hline 131 & 134274.83 & 13102.32 & 2445740.67 & 1193402.00 \\
\hline 132 & 134763.47 & 13154.44 & 2446207.85 & 1193553.96 \\
\hline 133 & 135234.46 & 13005.20 & 2446699.38 & 1193505.32 \\
\hline 134 & 135704.87 & 12841.41 & 2447193.35 & 1193442.32 \\
\hline 135 & 136179.15 & 12690.72 & 2447688.40 & 1193392.95 \\
\hline 136 & 136652.61 & 12539.84 & 2448182.69 & 1193343.22 \\
\hline 137 & 137071.59 & 12273.14 & 2448647.63 & 1193168.96 \\
\hline 138 & 137519.34 & 12051.23 & 2449131.46 & 1193044.45 \\
\hline 139 & 137986.63 & 11880.11 & 2449623.90 & 1192973.65 \\
\hline 140 & 138436.40 & 11668.72 & 2450107.52 & 1192859.86 \\
\hline 141 & 138838.49 & 11377.57 & 2450561.01 & 1192658.18 \\
\hline 142 & 139177.14 & 11013.33 & 2450967.55 & 1192371.91 \\
\hline 143 & 139572.36 & 10711.25 & 2451416.58 & 1192158.14 \\
\hline 144 & 140017.01 & 10500.85 & 2451895.01 & 1192044.26 \\
\hline 145 & 140510.13 & 10480.45 & 2452381.57 & 1192126.25 \\
\hline 146 & 140992.45 & 10586.43 & 2452831.43 & 1192329.63 \\
\hline 147 & 141483.13 & 10640.37 & 2453300.23 & 1192483.83 \\
\hline 148 & 141976.02 & 10697.42 & 2453770.56 & 1192641.54 \\
\hline 149 & 142465.74 & 10761.39 & 2454236.35 & 1192805.36 \\
\hline 150 & 142919.10 & 10572.13 & 2454718.92 & 1192713.98 \\
\hline 151 & 143158.48 & 10149.10 & 2455040.54 & 1192349.70 \\
\hline 152 & 142997.19 & 9710.22 & 2454973.53 & 1191887.05 \\
\hline 153 & 142752.67 & 9314.79 & 2454816.11 & 1191449.71 \\
\hline 154 & 143015.74 & 9050.77 & 2455128.03 & 1191245.86 \\
\hline 155 & 143429.80 & 9327.47 & 2455475.83 & 1191602.13 \\
\hline 156 & 143708.46 & 9730.20 & 2455665.12 & 1192053.68 \\
\hline 157 & 144008.45 & 9512.29 & 2456003.61 & 1191902.56 \\
\hline 158 & 144246.52 & 9071.81 & 2456327.57 & 1191520.94 \\
\hline 159 & 144063.72 & 8664.23 & 2456233.04 & 1191084.46 \\
\hline 160 & 143648.80 & 8434.17 & 2455874.76 & 1190773.62 \\
\hline 161 & 144047.06 & 8182.78 & 2456316.30 & 1190610.07 \\
\hline 162 & 144532.53 & 8251.53 & 2456776.96 & 1190777.72 \\
\hline 163 & 144963.14 & 8054.25 & 2457238.96 & 1190673.80 \\
\hline 164 & 145304.49 & 7703.46 & 2457645.41 & 1190401.26 \\
\hline 165 & 145561.36 & 7279.56 & 2457984.33 & 1190039.73 \\
\hline 166 & 145916.35 & 6940.67 & 2458401.66 & 1189781.65 \\
\hline 167 & 146365.34 & 6921.20 & 2458844.88 & 1189855.47 \\
\hline 168 & 146630.84 & 6515.51 & 2459188.49 & 1189513.54 \\
\hline
\end{tabular}




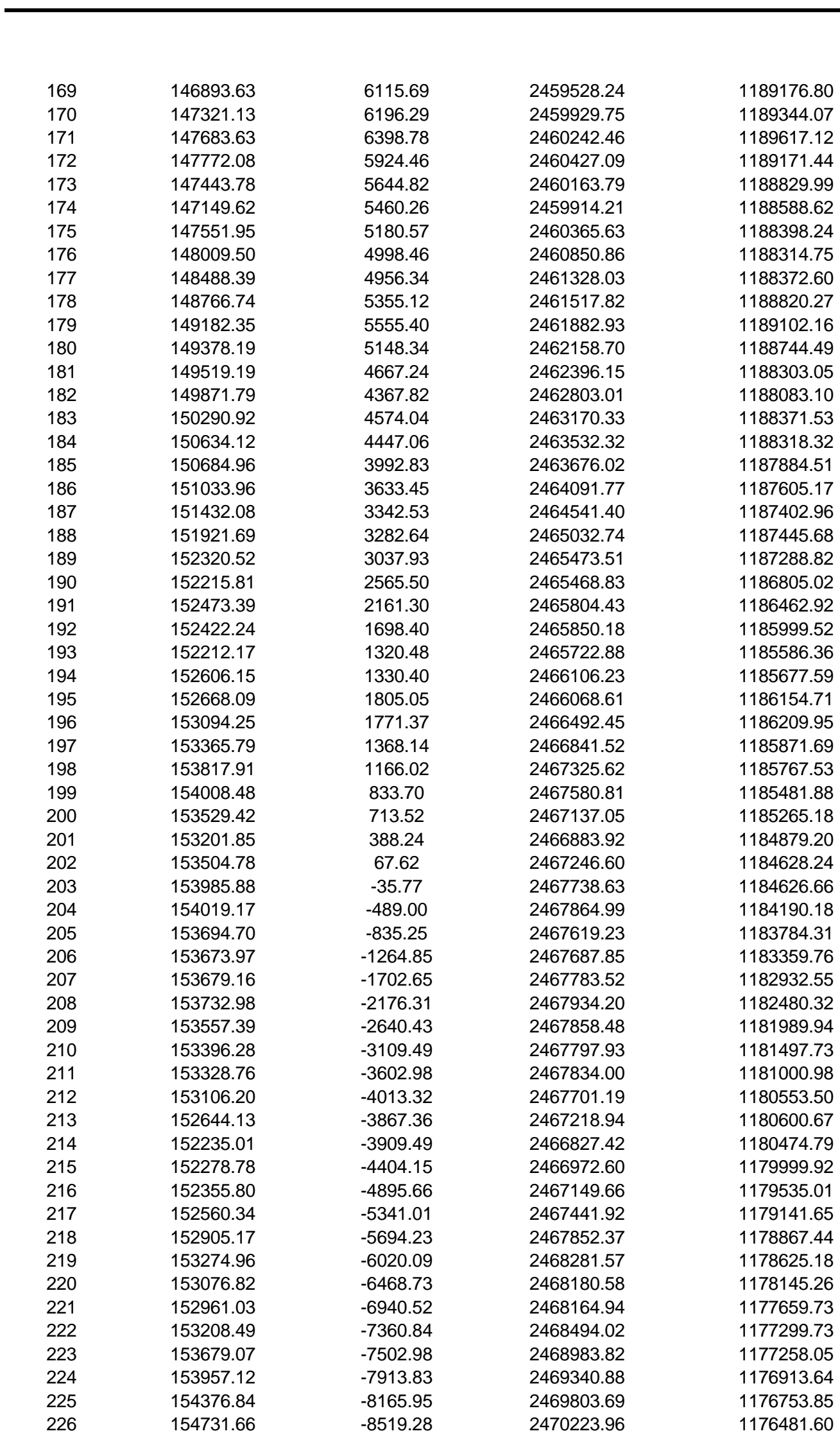


WSRC-TR-2002-00470

October 2002

\begin{tabular}{|c|c|c|c|c|}
\hline 227 & 154609.13 & -8939.79 & 2470191.11 & 1176044.84 \\
\hline 228 & 154390.32 & -9285.78 & 2470048.65 & 1175661.05 \\
\hline 229 & 154826.31 & -9405.68 & 2470500.03 & 1175633.98 \\
\hline 230 & 155037.73 & -9692.23 & 2470766.18 & 1175397.40 \\
\hline 231 & 155007.62 & -10151.02 & 2470831.68 & 1174942.29 \\
\hline 232 & 155142.85 & -10611.17 & 2471059.23 & 1174520.09 \\
\hline 233 & 154988.55 & -11072.16 & 2471003.69 & 1174037.13 \\
\hline 234 & 155366.65 & -11312.27 & 2471423.31 & 1173880.47 \\
\hline 235 & 155735.62 & -11629.55 & 2471849.99 & 1173646.42 \\
\hline 236 & 155889.02 & -11371.27 & 2471946.60 & 1173930.87 \\
\hline 237 & 155930.49 & -10911.85 & 2471892.08 & 1174388.95 \\
\hline 238 & 156388.67 & -11024.03 & 2472363.57 & 1174374.03 \\
\hline 239 & 156502.81 & -11453.39 & 2472564.13 & 1173977.58 \\
\hline 240 & 156165.01 & -11783.62 & 2472301.98 & 1173584.56 \\
\hline 241 & 155812.59 & -12085.40 & 2472019.65 & 1173216.36 \\
\hline 242 & 155391.20 & -12347.72 & 2471661.66 & 1172872.47 \\
\hline 243 & 155233.65 & -12798.29 & 2471600.78 & 1172399.02 \\
\hline 244 & 155225.47 & -13218.76 & 2471679.82 & 1171985.94 \\
\hline 245 & 155579.68 & -13532.54 & 2472091.32 & 1171752.26 \\
\hline 246 & 156056.48 & -13623.63 & 2472576.69 & 1171761.83 \\
\hline 247 & 156241.64 & -13228.93 & 2472676.15 & 1172186.34 \\
\hline 248 & 156604.74 & -13282.99 & 2473042.60 & 1172208.61 \\
\hline 249 & 156837.77 & -13721.80 & 2473361.44 & 1171827.51 \\
\hline 250 & 157275.87 & -13913.70 & 2473829.81 & 1171730.45 \\
\hline 251 & 157624.26 & -14123.08 & 2474214.03 & 1171597.72 \\
\hline 252 & 157733.62 & -14606.14 & 2474421.03 & 1171147.72 \\
\hline 253 & 157545.13 & -15055.50 & 2474329.64 & 1170669.03 \\
\hline 254 & 157537.42 & -15539.70 & 2474422.34 & 1170193.68 \\
\hline 255 & 157303.88 & -15978.69 & 2474284.72 & 1169715.81 \\
\hline 256 & 157195.12 & -16461.67 & 2474278.29 & 1169220.72 \\
\hline 257 & 157368.26 & -16914.70 & 2474541.50 & 1168813.30 \\
\hline 258 & 157545.12 & -17242.18 & 2474782.34 & 1168529.49 \\
\hline 259 & 157729.51 & -16838.06 & 2474879.10 & 1168963.08 \\
\hline 260 & 158132.27 & -16572.89 & 2475218.28 & 1169305.92 \\
\hline 261 & 158545.38 & -16591.81 & 2475626.39 & 1169372.94 \\
\hline 262 & 158536.44 & -17077.39 & 2475718.19 & 1168895.98 \\
\hline 263 & 158739.09 & -17532.90 & 2476010.78 & 1168492.24 \\
\hline 264 & 159130.84 & -17511.01 & 2476389.56 & 1168594.78 \\
\hline 265 & 159486.14 & -17316.86 & 2476697.00 & 1168858.31 \\
\hline 266 & 159930.11 & -17525.71 & 2477174.65 & 1168745.89 \\
\hline 267 & 160249.94 & -17893.39 & 2477563.73 & 1168452.36 \\
\hline 268 & 160526.32 & -18302.42 & 2477918.85 & 1168109.37 \\
\hline 269 & 160531.83 & -18783.52 & 2478023.87 & 1167639.77 \\
\hline 270 & 160171.05 & -19095.88 & 2477735.54 & 1167259.43 \\
\hline 271 & 160285.16 & -19514.55 & 2477933.89 & 1166873.40 \\
\hline 272 & 160468.08 & -19924.79 & 2478197.82 & 1166509.86 \\
\hline 273 & 160828.25 & -20151.71 & 2478597.24 & 1166362.42 \\
\hline 274 & 160650.54 & -20615.66 & 2478519.43 & 1165871.64 \\
\hline 275 & 160292.74 & -20952.89 & 2478239.16 & 1165467.57 \\
\hline 276 & 160194.17 & -21341.76 & 2478223.24 & 1165066.66 \\
\hline 277 & 160425.79 & -21779.42 & 2478540.50 & 1164686.36 \\
\hline 278 & 160017.84 & -21872.06 & 2478160.51 & 1164511.24 \\
\hline 279 & 160012.32 & -22216.31 & 2478226.39 & 1164173.24 \\
\hline 280 & 160142.52 & -22690.69 & 2478452.04 & 1163736.02 \\
\hline 281 & 160226.14 & -23150.25 & 2478629.03 & 1163303.65 \\
\hline 282 & 159917.27 & -23511.13 & 2478401.52 & 1162886.55 \\
\hline 283 & 160345.67 & -23674.83 & 2478854.63 & 1162815.07 \\
\hline 284 & 160258.85 & -24034.00 & 2478844.05 & 1162445.64 \\
\hline
\end{tabular}




\begin{tabular}{|c|c|c|c|c|}
\hline 285 & 159832.08 & -23860.04 & 2478390.41 & 1162527.48 \\
\hline 286 & 159616.95 & -23997.48 & 2478208.37 & 1162348.45 \\
\hline 287 & 159941.01 & -24366.31 & 2478601.85 & 1162054.64 \\
\hline 288 & 160408.69 & -24490.64 & 2479085.24 & 1162029.83 \\
\hline 289 & 160895.62 & -24372.88 & 2479537.33 & 1162245.90 \\
\hline 290 & 161085.81 & -24652.52 & 2479781.35 & 1162011.65 \\
\hline 291 & 160853.43 & -25092.97 & 2479645.18 & 1161532.52 \\
\hline 292 & 160369.73 & -25121.09 & 2479177.68 & 1161404.83 \\
\hline 293 & 159874.11 & -25193.66 & 2478707.71 & 1161231.19 \\
\hline 294 & 159494.42 & -25420.56 & 2478383.16 & 1160930.52 \\
\hline 295 & 159835.59 & -25677.91 & 2478770.31 & 1160749.34 \\
\hline 296 & 160324.45 & -25616.57 & 2479235.98 & 1160910.61 \\
\hline 297 & 160791.24 & -25769.63 & 2479724.46 & 1160857.50 \\
\hline 298 & 161199.43 & -26026.59 & 2480177.12 & 1160690.58 \\
\hline 299 & 161357.33 & -26425.13 & 2480414.17 & 1160333.29 \\
\hline 300 & 161189.82 & -26892.58 & 2480347.06 & 1159841.17 \\
\hline 301 & 160939.03 & -27298.76 & 2480185.77 & 1159391.75 \\
\hline 302 & 160633.17 & -27673.16 & 2479964.00 & 1158962.02 \\
\hline 303 & 160983.52 & -27881.18 & 2480349.93 & 1158831.02 \\
\hline 304 & 161468.43 & -27755.47 & 2480798.42 & 1159054.47 \\
\hline 305 & 161960.80 & -27781.08 & 2481285.55 & 1159131.39 \\
\hline 306 & 162007.92 & -28261.32 & 2481431.13 & 1158671.19 \\
\hline 307 & 161817.23 & -28712.09 & 2481337.89 & 1158190.58 \\
\hline 308 & 161352.34 & -28869.40 & 2480915.53 & 1157940.34 \\
\hline 309 & 161270.42 & -29268.45 & 2480918.01 & 1157532.87 \\
\hline 310 & 161243.03 & -29653.88 & 2480971.04 & 1157150.01 \\
\hline 311 & 160778.80 & -29575.94 & 2480500.60 & 1157130.13 \\
\hline 312 & 160904.43 & -30007.41 & 2480712.90 & 1156733.91 \\
\hline 313 & 160776.41 & -30464.76 & 2480682.35 & 1156259.83 \\
\hline 314 & 160571.00 & -30880.90 & 2480567.51 & 1155810.04 \\
\hline 315 & 160799.05 & -31284.26 & 2480874.23 & 1155462.54 \\
\hline 316 & 161060.55 & -31678.44 & 2481211.78 & 1155130.94 \\
\hline 317 & 160664.81 & -31840.88 & 2480858.14 & 1154890.00 \\
\hline 318 & 160356.31 & -32139.97 & 2480618.19 & 1154533.42 \\
\hline 319 & 160190.19 & -32571.20 & 2480544.92 & 1154076.99 \\
\hline 320 & 159888.65 & -32966.92 & 2480331.78 & 1153627.27 \\
\hline 321 & 160286.35 & -33122.15 & 2480753.14 & 1153557.71 \\
\hline 322 & 160605.33 & -33433.67 & 2481129.83 & 1153318.91 \\
\hline 323 & 160949.57 & -33709.51 & 2481523.86 & 1153120.25 \\
\hline 324 & 161413.63 & -33798.95 & 2481996.55 & 1153128.84 \\
\hline 325 & 161769.07 & -34128.49 & 2482412.65 & 1152879.96 \\
\hline 326 & 161899.16 & -34568.66 & 2482631.15 & 1152476.13 \\
\hline 327 & 161938.09 & -34997.87 & 2482758.16 & 1152064.14 \\
\hline 328 & 162296.24 & -35315.13 & 2483174.38 & 1151827.83 \\
\hline 329 & 162327.57 & -35802.22 & 2483305.95 & 1151357.62 \\
\hline 330 & 162147.52 & -36267.97 & 2483226.21 & 1150864.50 \\
\hline 331 & 162052.73 & -36754.52 & 2483234.23 & 1150368.68 \\
\hline 332 & 162332.93 & -37149.83 & 2483590.35 & 1150039.84 \\
\hline 333 & 162704.62 & -37456.34 & 2484017.62 & 1149816.85 \\
\hline 334 & 162598.73 & -37904.80 & 2484006.89 & 1149356.00 \\
\hline 335 & 162846.28 & -38270.93 & 2484325.01 & 1149048.96 \\
\hline 336 & 163080.19 & -38647.20 & 2484631.90 & 1148729.15 \\
\hline 337 & 162719.82 & -38973.52 & 2484346.80 & 1148335.12 \\
\hline 338 & 162360.87 & -39307.37 & 2484064.65 & 1147934.01 \\
\hline 339 & 161946.02 & -39578.43 & 2483714.77 & 1147582.78 \\
\hline 340 & 161452.55 & -39626.28 & 2483241.71 & 1147433.72 \\
\hline 341 & 161283.66 & -39976.42 & 2483148.94 & 1147056.04 \\
\hline 342 & 160962.53 & -40201.63 & 2482881.29 & 1146769.09 \\
\hline
\end{tabular}


WSRC-TR-2002-00470

October 2002

\begin{tabular}{|c|c|c|c|c|}
\hline 343 & 160696.30 & -40589.72 & 2482701.11 & 1146334.11 \\
\hline 344 & 160698.13 & -41083.51 & 2482805.18 & 1145851.19 \\
\hline 345 & 161035.24 & -41432.41 & 2483207.40 & 1145579.53 \\
\hline 346 & 161268.53 & -41830.13 & 2483518.12 & 1145238.58 \\
\hline 347 & 161336.95 & -42293.91 & 2483681.17 & 1144798.83 \\
\hline 348 & 161738.94 & -42523.13 & 2484122.11 & 1144657.75 \\
\hline 349 & 161913.64 & -42983.76 & 2484388.52 & 1144243.09 \\
\hline 350 & 162282.77 & -43318.58 & 2484819.19 & 1143991.85 \\
\hline 351 & 162652.69 & -43653.04 & 2485250.55 & 1143741.11 \\
\hline 352 & 162974.27 & -44028.80 & 2485643.16 & 1143439.95 \\
\hline 353 & 163219.25 & -44462.65 & 2485972.83 & 1143066.06 \\
\hline 354 & 163485.21 & -44881.97 & 2486320.02 & 1142710.74 \\
\hline 355 & 163863.08 & -45209.26 & 2486757.69 & 1142468.68 \\
\hline 356 & 164269.25 & -45495.73 & 2487214.61 & 1142272.44 \\
\hline 357 & 164719.93 & -45436.80 & 2487643.52 & 1142423.50 \\
\hline 358 & 165100.18 & -45213.81 & 2487969.51 & 1142720.55 \\
\hline 359 & 165299.95 & -45586.25 & 2488242.22 & 1142397.41 \\
\hline 360 & 165310.58 & -46026.13 & 2488343.77 & 1141969.05 \\
\hline 361 & 165783.80 & -46118.61 & 2488826.12 & 1141976.59 \\
\hline 362 & 165677.34 & -46541.56 & 2488809.57 & 1141540.54 \\
\hline 363 & 165597.03 & -46898.26 & 2488804.87 & 1141174.76 \\
\hline 364 & 166004.25 & -46700.18 & 2489162.41 & 1141453.03 \\
\hline 365 & 166257.11 & -46298.67 & 2489326.72 & 1141898.43 \\
\hline 366 & 166690.90 & -46516.83 & 2489796.52 & 1141774.79 \\
\hline 367 & 166613.74 & -46916.69 & 2489803.86 & 1141367.41 \\
\hline 368 & 166355.72 & -47264.49 & 2489623.39 & 1140973.50 \\
\hline 369 & 166740.14 & -47561.53 & 2490061.22 & 1140762.43 \\
\hline 370 & 167214.74 & -47710.28 & 2490556.60 & 1140715.19 \\
\hline 371 & 167658.85 & -47931.38 & 2491037.13 & 1140590.81 \\
\hline 372 & 168100.50 & -48160.15 & 2491516.84 & 1140458.41 \\
\hline 373 & 168567.96 & -48333.02 & 2492010.23 & 1140386.10 \\
\hline 374 & 169006.66 & -48573.15 & 2492489.41 & 1140241.98 \\
\hline 375 & 169462.22 & -48758.75 & 2492973.80 & 1140154.73 \\
\hline 376 & 169952.54 & -48823.49 & 2493467.16 & 1140192.99 \\
\hline 377 & 170443.38 & -48731.87 & 2493928.61 & 1140384.42 \\
\hline 378 & 170937.61 & -48675.44 & 2494400.68 & 1140542.10 \\
\hline 379 & 171423.86 & -48570.52 & 2494854.88 & 1140745.60 \\
\hline 380 & 171904.79 & -48443.26 & 2495299.25 & 1140969.86 \\
\hline 381 & 172245.21 & -48734.44 & 2495692.82 & 1140755.43 \\
\hline 382 & 172026.16 & -49171.68 & 2495569.05 & 1140282.03 \\
\hline 383 & 172189.87 & -49515.28 & 2495800.53 & 1139979.65 \\
\hline 384 & 172589.99 & -49249.44 & 2496137.06 & 1140322.81 \\
\hline 385 & 172510.07 & -48777.31 & 2495960.96 & 1140768.38 \\
\hline 386 & 172671.86 & -48517.52 & 2496065.46 & 1141056.20 \\
\hline 387 & 172875.15 & -48947.49 & 2496353.59 & 1140677.49 \\
\hline 388 & 172818.82 & -49430.12 & 2496398.51 & 1140193.39 \\
\hline 389 & 172940.79 & -49768.31 & 2496588.02 & 1139887.66 \\
\hline 390 & 173264.28 & -49439.64 & 2496836.51 & 1140276.43 \\
\hline 391 & 173528.63 & -49172.31 & 2497039.84 & 1140592.91 \\
\hline 392 & 173796.57 & -49564.09 & 2497383.34 & 1140264.98 \\
\hline 393 & 173566.32 & -49980.80 & 2497244.36 & 1139809.35 \\
\hline 394 & 173848.44 & -50333.61 & 2497593.66 & 1139522.50 \\
\hline 395 & 174155.12 & -50626.04 & 2497954.48 & 1139299.85 \\
\hline 396 & 174076.73 & -51106.35 & 2497977.34 & 1138813.44 \\
\hline 397 & 173964.04 & -51355.24 & 2497918.64 & 1138546.46 \\
\hline 398 & 174066.32 & -51589.38 & 2498067.31 & 1138338.47 \\
\hline 399 & 174347.60 & -51976.94 & 2498423.00 & 1138017.43 \\
\hline 400 & 174729.88 & -52266.22 & 2498857.18 & 1137813.54 \\
\hline
\end{tabular}




\begin{tabular}{|c|c|c|c|c|}
\hline 401 & 175126.42 & -52558.15 & 2499305.87 & 1137610.00 \\
\hline 402 & 175530.86 & -52846.56 & 2499761.57 & 1137411.55 \\
\hline 403 & 175907.70 & -53166.51 & 2500196.80 & 1137176.51 \\
\hline 404 & 176310.27 & -53457.76 & 2500651.26 & 1136974.89 \\
\hline 405 & 176788.92 & -53426.33 & 2501113.27 & 1137104.92 \\
\hline 406 & 177248.94 & -53562.91 & 2501591.90 & 1137066.64 \\
\hline 407 & 177703.43 & -53536.40 & 2502031.29 & 1137186.84 \\
\hline 408 & 178115.12 & -53727.11 & 2502473.84 & 1137085.54 \\
\hline 409 & 178067.21 & -54168.33 & 2502518.44 & 1136643.71 \\
\hline 410 & 177603.69 & -54289.77 & 2502089.90 & 1136428.70 \\
\hline 411 & 177580.57 & -54767.00 & 2502166.24 & 1135956.75 \\
\hline 412 & 177928.68 & -55115.90 & 2502579.37 & 1135687.42 \\
\hline 413 & 178020.47 & -55595.83 & 2502768.76 & 1135236.66 \\
\hline 414 & 178409.86 & -55889.05 & 2503210.75 & 1135030.39 \\
\hline 415 & 178773.34 & -56229.18 & 2503637.11 & 1134772.83 \\
\hline 416 & 179102.19 & -56600.32 & 2504036.00 & 1134477.73 \\
\hline 417 & 179370.53 & -57025.59 & 2504386.89 & 1134117.08 \\
\hline 418 & 179712.19 & -57379.83 & 2504794.83 & 1133841.18 \\
\hline 419 & 180073.43 & -57718.55 & 2505218.71 & 1133584.54 \\
\hline 420 & 180426.28 & -58078.26 & 2505638.74 & 1133305.61 \\
\hline 421 & 180750.31 & -58456.79 & 2506034.47 & 1133002.29 \\
\hline 422 & 181094.14 & -58819.52 & 2506446.30 & 1132718.52 \\
\hline 423 & 181488.40 & -59126.56 & 2506895.96 & 1132499.74 \\
\hline 424 & 181939.21 & -59311.10 & 2507375.55 & 1132412.62 \\
\hline 425 & 182396.22 & -59360.12 & 2507833.10 & 1132459.45 \\
\hline 426 & 182320.54 & -59788.55 & 2507847.91 & 1132024.35 \\
\hline 427 & 181876.87 & -59990.35 & 2507455.45 & 1131734.74 \\
\hline 428 & 182045.09 & -60396.33 & 2507704.35 & 1131372.21 \\
\hline 429 & 181761.29 & -60687.67 & 2507486.97 & 1131028.13 \\
\hline 430 & 181360.00 & -60479.25 & 2507050.89 & 1131148.91 \\
\hline 431 & 181095.07 & -60593.15 & 2506815.17 & 1130982.45 \\
\hline 432 & 180773.42 & -60794.74 & 2506542.11 & 1130718.37 \\
\hline 433 & 180618.45 & -61264.69 & 2506487.90 & 1130226.16 \\
\hline 434 & 181054.42 & -61384.71 & 2506939.59 & 1130199.11 \\
\hline 435 & 181279.46 & -61787.93 & 2507243.55 & 1129851.05 \\
\hline 436 & 181012.25 & -62167.35 & 2507060.68 & 1129424.18 \\
\hline 437 & 180576.30 & -62406.89 & 2506683.59 & 1129099.23 \\
\hline 438 & 180540.55 & -62833.92 & 2506737.18 & 1128673.76 \\
\hline 439 & 180685.56 & -63312.07 & 2506978.34 & 1128235.75 \\
\hline 440 & 180747.20 & -63798.83 & 2507139.67 & 1127772.01 \\
\hline 441 & 180925.11 & -64212.36 & 2507399.63 & 1127404.07 \\
\hline 442 & 181229.97 & -64611.75 & 2507780.96 & 1127076.32 \\
\hline 443 & 181509.27 & -65025.15 & 2508140.16 & 1126729.54 \\
\hline 444 & 181830.90 & -65406.72 & 2508534.21 & 1126422.71 \\
\hline 445 & 182171.52 & -65773.53 & 2508943.79 & 1126134.28 \\
\hline 446 & 182413.48 & -66211.24 & 2509271.50 & 1125755.96 \\
\hline 447 & 182706.39 & -66610.26 & 2509641.06 & 1125426.08 \\
\hline 448 & 183032.88 & -66985.86 & 2510038.63 & 1125126.11 \\
\hline 449 & 183295.61 & -67406.00 & 2510383.04 & 1124769.30 \\
\hline 450 & 183343.61 & -67893.55 & 2510531.20 & 1124301.93 \\
\hline 451 & 183208.83 & -68374.11 & 2510498.96 & 1123803.47 \\
\hline 452 & 182960.69 & -68799.69 & 2510344.33 & 1123335.32 \\
\hline 453 & 183350.88 & -69079.49 & 2510784.41 & 1123142.34 \\
\hline 454 & 183637.38 & -69485.65 & 2511149.19 & 1122804.15 \\
\hline 455 & 184020.42 & -69800.41 & 2511589.53 & 1122575.46 \\
\hline 456 & 184216.06 & -70205.23 & 2511865.09 & 1122219.72 \\
\hline 457 & 184030.76 & -70476.28 & 2511739.92 & 1121915.89 \\
\hline 458 & 184164.95 & -70787.23 & 2511935.83 & 1121639.30 \\
\hline
\end{tabular}


WSRC-TR-2002-00470

October 2002

\begin{tabular}{|c|c|c|c|c|}
\hline 459 & 184267.76 & -71090.43 & 2512099.41 & 1121363.78 \\
\hline 460 & 184686.01 & -71345.15 & 2512561.78 & 1121201.19 \\
\hline 461 & 184867.17 & -71766.53 & 2512826.59 & 1120826.22 \\
\hline 462 & 185008.98 & -72120.62 & 2513038.93 & 1120508.97 \\
\hline 463 & 185430.34 & -72321.50 & 2513493.17 & 1120399.74 \\
\hline 464 & 185368.20 & -72714.92 & 2513513.98 & 1120001.65 \\
\hline 465 & 185521.42 & -72856.77 & 2513693.43 & 1119894.56 \\
\hline 466 & 185819.60 & -72669.40 & 2513946.50 & 1120139.91 \\
\hline 467 & 186115.71 & -72517.22 & 2514204.83 & 1120350.36 \\
\hline 468 & 186038.08 & -72198.34 & 2514062.64 & 1120646.46 \\
\hline 469 & 186418.67 & -71897.02 & 2514372.72 & 1121020.47 \\
\hline 470 & 186768.15 & -72173.62 & 2514772.31 & 1120822.20 \\
\hline 471 & 186962.77 & -72621.93 & 2515055.91 & 1120423.66 \\
\hline 472 & 187311.66 & -72895.27 & 2515454.24 & 1120228.45 \\
\hline 473 & 187709.71 & -72951.67 & 2515855.68 & 1120255.86 \\
\hline 474 & 187919.62 & -73371.01 & 2516148.25 & 1119888.86 \\
\hline 475 & 187714.61 & -73745.79 & 2516025.32 & 1119479.36 \\
\hline 476 & 187388.46 & -74081.21 & 2515775.61 & 1119083.24 \\
\hline 477 & 187089.23 & -74461.16 & 2515561.50 & 1118649.12 \\
\hline 478 & 187242.43 & -74904.29 & 2515803.49 & 1118247.04 \\
\hline 479 & 187496.08 & -75085.49 & 2516089.45 & 1118122.28 \\
\hline 480 & 187680.35 & -75298.78 & 2516314.15 & 1117951.69 \\
\hline 481 & 187691.72 & -75783.32 & 2516425.86 & 1117479.63 \\
\hline 482 & 187796.26 & -76263.72 & 2516627.95 & 1117030.95 \\
\hline 483 & 187514.62 & -76639.54 & 2516430.20 & 1116604.52 \\
\hline 484 & 187905.97 & -76832.13 & 2516853.37 & 1116497.18 \\
\hline 485 & 188280.78 & -76632.33 & 2517178.88 & 1116770.62 \\
\hline 486 & 188519.92 & -77054.77 & 2517500.73 & 1116406.64 \\
\hline 487 & 188506.43 & -77548.63 & 2517590.05 & 1115920.28 \\
\hline 488 & 188399.77 & -78039.37 & 2517587.49 & 1115417.62 \\
\hline 489 & 188587.14 & -78465.95 & 2517859.52 & 1115038.84 \\
\hline 490 & 188769.34 & -78925.66 & 2518133.36 & 1114626.54 \\
\hline 491 & 188633.68 & -79381.20 & 2518095.10 & 1114152.31 \\
\hline 492 & 188166.73 & -79537.52 & 2517670.33 & 1113902.31 \\
\hline 493 & 188164.63 & -79956.84 & 2517755.33 & 1113491.29 \\
\hline 494 & 188429.66 & -80349.25 & 2518096.31 & 1113162.06 \\
\hline 495 & 188050.72 & -80416.53 & 2517739.22 & 1113017.52 \\
\hline 496 & 187663.49 & -80560.42 & 2517389.92 & 1112796.23 \\
\hline 497 & 187557.85 & -81017.01 & 2517381.27 & 1112327.21 \\
\hline 498 & 187257.58 & -81265.48 & 2517138.83 & 1112021.57 \\
\hline 499 & 187020.46 & -81624.74 & 2516981.22 & 1111620.56 \\
\hline 500 & 186568.00 & -81752.06 & 2516564.59 & 1111401.96 \\
\hline 501 & 186241.84 & -81964.29 & 2516289.28 & 1111126.44 \\
\hline 502 & 186387.80 & -82389.84 & 2516520.54 & 1110740.04 \\
\hline 503 & 186140.33 & -82726.32 & 2516348.05 & 1110359.18 \\
\hline 504 & 186291.46 & -83162.22 & 2516586.54 & 1109963.70 \\
\hline 505 & 185862.80 & -83378.58 & 2516211.70 & 1109662.85 \\
\hline 506 & 185430.00 & -83622.90 & 2515838.60 & 1109333.76 \\
\hline 507 & 185370.95 & -84099.17 & 2515879.64 & 1108855.12 \\
\hline 508 & 185612.16 & -84512.30 & 2516201.60 & 1108500.64 \\
\hline 509 & 185992.13 & -84830.19 & 2516639.67 & 1108268.22 \\
\hline 510 & 186412.47 & -85095.28 & 2517106.33 & 1108095.90 \\
\hline 511 & 186570.86 & -85565.35 & 2517359.01 & 1107668.46 \\
\hline 512 & 186361.68 & -86005.38 & 2517245.52 & 1107194.13 \\
\hline 513 & 186430.00 & -86304.61 & 2517374.54 & 1106915.29 \\
\hline 514 & 186868.99 & -86514.82 & 2517848.07 & 1106800.57 \\
\hline 515 & 187124.70 & -86927.96 & 2518184.26 & 1106449.08 \\
\hline 516 & 187264.45 & -87387.68 & 2518416.56 & 1106027.90 \\
\hline
\end{tabular}




\begin{tabular}{|c|c|c|c|c|}
\hline 517 & 187705.52 & -87615.50 & 2518895.78 & 1105896.38 \\
\hline 518 & 188044.07 & -87966.52 & 2519300.20 & 1105622.92 \\
\hline 519 & 188257.34 & -88414.86 & 2519602.13 & 1105228.15 \\
\hline 520 & 188456.11 & -88858.02 & 2519888.80 & 1104835.43 \\
\hline 521 & 188620.72 & -89291.87 & 2520140.08 & 1104444.75 \\
\hline 522 & 188639.34 & -89564.18 & 2520214.86 & 1104181.94 \\
\hline 523 & 188679.70 & -89854.10 & 2520314.59 & 1103906.40 \\
\hline 524 & 188886.76 & -90227.89 & 2520594.98 & 1103583.34 \\
\hline 525 & 188791.48 & -90710.39 & 2520601.85 & 1103091.04 \\
\hline 526 & 188670.31 & -91160.78 & 2520576.71 & 1102624.79 \\
\hline 527 & 188592.63 & -91546.99 & 2520580.83 & 1102230.44 \\
\hline 528 & 188252.99 & -91907.30 & 2520323.03 & 1101807.06 \\
\hline 529 & 187909.21 & -92250.00 & 2520057.51 & 1101400.06 \\
\hline 530 & 187939.22 & -92662.10 & 2520172.46 & 1101002.70 \\
\hline 531 & 188398.93 & -92847.47 & 2520661.17 & 1100916.62 \\
\hline 532 & 188475.53 & -93314.45 & 2520833.15 & 1100475.19 \\
\hline 533 & 188581.11 & -93802.14 & 2521037.82 & 1100019.49 \\
\hline 534 & 189007.70 & -93965.76 & 2521489.58 & 1099947.82 \\
\hline 535 & 189404.26 & -94170.80 & 2521920.53 & 1099829.37 \\
\hline 536 & 189238.57 & -94563.22 & 2521839.75 & 1099410.64 \\
\hline 537 & 188868.90 & -94869.69 & 2521541.35 & 1099033.73 \\
\hline 538 & 188785.00 & -95352.19 & 2521559.38 & 1098543.75 \\
\hline 539 & 188694.89 & -95840.92 & 2521572.61 & 1098046.40 \\
\hline 540 & 188965.12 & -96202.30 & 2521912.31 & 1097748.58 \\
\hline 541 & 189256.06 & -96604.07 & 2522280.68 & 1097415.51 \\
\hline 542 & 189264.32 & -97096.94 & 2522391.12 & 1096934.50 \\
\hline 543 & 189127.61 & -97577.38 & 2522357.00 & 1096435.57 \\
\hline 544 & 189154.51 & -98023.66 & 2522476.02 & 1096004.07 \\
\hline 545 & 189606.99 & -98258.73 & 2522968.01 & 1095867.80 \\
\hline 546 & 190072.93 & -98419.25 & 2523457.69 & 1095807.35 \\
\hline 547 & 190544.06 & -98522.82 & 2523940.63 & 1095803.76 \\
\hline 548 & 191046.26 & -98481.43 & 2524423.89 & 1095948.60 \\
\hline 549 & 191455.25 & -98717.53 & 2524873.50 & 1095802.31 \\
\hline 550 & 191666.46 & -99155.54 & 2525171.34 & 1095417.19 \\
\hline 551 & 143300.15 & 43768.97 & 2448228.98 & 1225256.64 \\
\hline 552 & 143091.06 & 43421.27 & 2448096.42 & 1224873.47 \\
\hline 553 & 142805.39 & 43045.62 & 2447894.76 & 1224447.15 \\
\hline 554 & 142570.43 & 42645.13 & 2447747.80 & 1224007.03 \\
\hline 555 & 142563.13 & 42155.62 & 2447841.84 & 1223526.92 \\
\hline 556 & 142221.58 & 41800.68 & 2447581.24 & 1223109.29 \\
\hline 557 & 141783.79 & 41581.33 & 2447198.53 & 1222804.34 \\
\hline 558 & 141472.24 & 41218.11 & 2446968.99 & 1222384.82 \\
\hline 559 & 141204.16 & 40804.17 & 2446792.41 & 1221924.69 \\
\hline 560 & 141253.78 & 40339.49 & 2446936.97 & 1221480.61 \\
\hline 561 & 141220.62 & 39860.33 & 2447003.56 & 1221005.25 \\
\hline 562 & 141228.85 & 39383.23 & 2447110.21 & 1220540.46 \\
\hline 563 & 141193.61 & 38889.58 & 2447177.77 & 1220050.50 \\
\hline 564 & 141014.52 & 38441.48 & 2447095.27 & 1219575.35 \\
\hline 565 & 140839.57 & 37998.56 & 2447015.74 & 1219106.11 \\
\hline 566 & 140539.41 & 37700.54 & 2446783.84 & 1218752.67 \\
\hline 567 & 140344.80 & 37261.75 & 2446684.23 & 1218283.42 \\
\hline 568 & 140348.88 & 36778.45 & 2446788.10 & 1217811.68 \\
\hline 569 & 140142.88 & 36339.67 & 2446677.36 & 1217340.07 \\
\hline 570 & 140123.17 & 35846.01 & 2446760.09 & 1216853.29 \\
\hline 571 & 139894.41 & 35546.95 & 2446598.22 & 1216513.59 \\
\hline 572 & 139675.16 & 35244.74 & 2446446.29 & 1216172.78 \\
\hline 573 & 139368.80 & 34974.67 & 2446202.52 & 1215845.37 \\
\hline 574 & 139117.27 & 34664.22 & 2446020.73 & 1215489.83 \\
\hline
\end{tabular}


WSRC-TR-2002-00470

October 2002

\begin{tabular}{|c|c|c|c|c|}
\hline 575 & 138986.82 & 34210.94 & 2445986.84 & 1215019.64 \\
\hline 576 & 138650.44 & 33950.18 & 2445711.80 & 1214695.15 \\
\hline 577 & 138403.08 & 33858.10 & 2445488.95 & 1214553.99 \\
\hline 578 & 138027.38 & 33620.12 & 2445170.74 & 1214243.64 \\
\hline 579 & 137637.23 & 33701.92 & 2444772.33 & 1214243.02 \\
\hline 580 & 137194.28 & 33664.72 & 2444346.87 & 1214115.11 \\
\hline 581 & 136793.77 & 33758.95 & 2443935.77 & 1214124.50 \\
\hline 582 & 136521.56 & 33481.62 & 2443726.89 & 1213797.07 \\
\hline 583 & 136156.21 & 33346.09 & 2443397.63 & 1213589.05 \\
\hline 584 & 135698.76 & 33187.81 & 2442983.01 & 1213339.76 \\
\hline 585 & 135489.65 & 32764.55 & 2442865.98 & 1212882.67 \\
\hline 586 & 135380.93 & 32298.86 & 2442855.88 & 1212404.81 \\
\hline 587 & 135226.67 & 31867.31 & 2442794.19 & 1211950.94 \\
\hline 588 & 135085.93 & 31516.01 & 2442729.15 & 1211578.33 \\
\hline 589 & 134827.15 & 31146.58 & 2442552.42 & 1211163.60 \\
\hline 590 & 134649.10 & 30818.53 & 2442446.08 & 1210806.02 \\
\hline 591 & 134532.10 & 30417.00 & 2442414.62 & 1210389.18 \\
\hline 592 & 134300.25 & 30191.41 & 2442234.50 & 1210120.69 \\
\hline 593 & 133981.45 & 29883.05 & 2441986.45 & 1209753.27 \\
\hline 594 & 133949.34 & 29642.95 & 2442004.65 & 1209511.84 \\
\hline 595 & 133905.81 & 29203.12 & 2442052.95 & 1209072.73 \\
\hline 596 & 133819.86 & 28846.08 & 2442042.67 & 1208705.82 \\
\hline 597 & 133487.61 & 28512.88 & 2441786.58 & 1208311.33 \\
\hline 598 & 133091.21 & 28325.62 & 2441437.62 & 1208046.30 \\
\hline 599 & 132708.24 & 28030.72 & 2441124.03 & 1207678.79 \\
\hline 600 & 132442.21 & 27613.68 & 2440950.01 & 1207216.00 \\
\hline 601 & 132174.13 & 27391.21 & 2440733.81 & 1206943.05 \\
\hline 602 & 131786.02 & 27266.04 & 2440380.11 & 1206740.47 \\
\hline 603 & 131461.07 & 27418.22 & 2440030.89 & 1206822.17 \\
\hline 604 & 131183.67 & 27088.12 & 2439827.79 & 1206442.04 \\
\hline 605 & 130905.24 & 26830.47 & 2439608.72 & 1206132.55 \\
\hline 606 & 130500.57 & 26737.38 & 2439232.20 & 1205957.93 \\
\hline 607 & 130168.33 & 26557.36 & 2438944.47 & 1205713.24 \\
\hline 608 & 129750.20 & 26404.25 & 2438567.17 & 1205477.14 \\
\hline 609 & 129315.52 & 26349.46 & 2438153.39 & 1205333.78 \\
\hline 610 & 128956.40 & 26311.23 & 2437810.08 & 1205222.21 \\
\hline 611 & 128637.65 & 26311.27 & 2437498.33 & 1205156.42 \\
\hline 612 & 128236.11 & 26377.57 & 2437091.95 & 1205138.33 \\
\hline 613 & 127787.96 & 26198.60 & 2436690.63 & 1204870.74 \\
\hline 614 & 127408.12 & 26040.31 & 2436351.85 & 1204637.49 \\
\hline 615 & 127401.85 & 25660.50 & 2436424.16 & 1204264.75 \\
\hline 616 & 127609.83 & 25416.23 & 2436678.01 & 1204068.81 \\
\hline 617 & 127768.11 & 24979.47 & 2436923.00 & 1203674.35 \\
\hline 618 & 140941.89 & 32119.74 & 2448330.79 & 1213378.81 \\
\hline 619 & 141062.00 & 32597.87 & 2448349.42 & 1213871.17 \\
\hline 620 & 141222.47 & 33069.77 & 2448408.79 & 1214365.79 \\
\hline 621 & 141323.94 & 33514.78 & 2448416.05 & 1214821.91 \\
\hline 622 & 141320.89 & 33998.09 & 2448313.17 & 1215293.88 \\
\hline 623 & 141074.63 & 34431.75 & 2447982.74 & 1215667.03 \\
\hline 624 & 140815.94 & 34846.78 & 2447644.02 & 1216019.40 \\
\hline 625 & 140589.35 & 35272.16 & 2447334.53 & 1216388.51 \\
\hline 626 & 140195.07 & 35526.80 & 2446896.37 & 1216556.02 \\
\hline 627 & 134182.44 & 39369.36 & 2440223.25 & 1219070.98 \\
\hline 628 & 133832.60 & 39059.97 & 2439945.10 & 1218696.19 \\
\hline 629 & 133502.43 & 38691.59 & 2439698.35 & 1218267.78 \\
\hline 630 & 133248.82 & 38272.48 & 2439536.95 & 1217805.60 \\
\hline 631 & 133156.66 & 37779.88 & 2439548.58 & 1217304.89 \\
\hline 632 & 133043.79 & 37291.42 & 2439539.12 & 1216803.95 \\
\hline
\end{tabular}




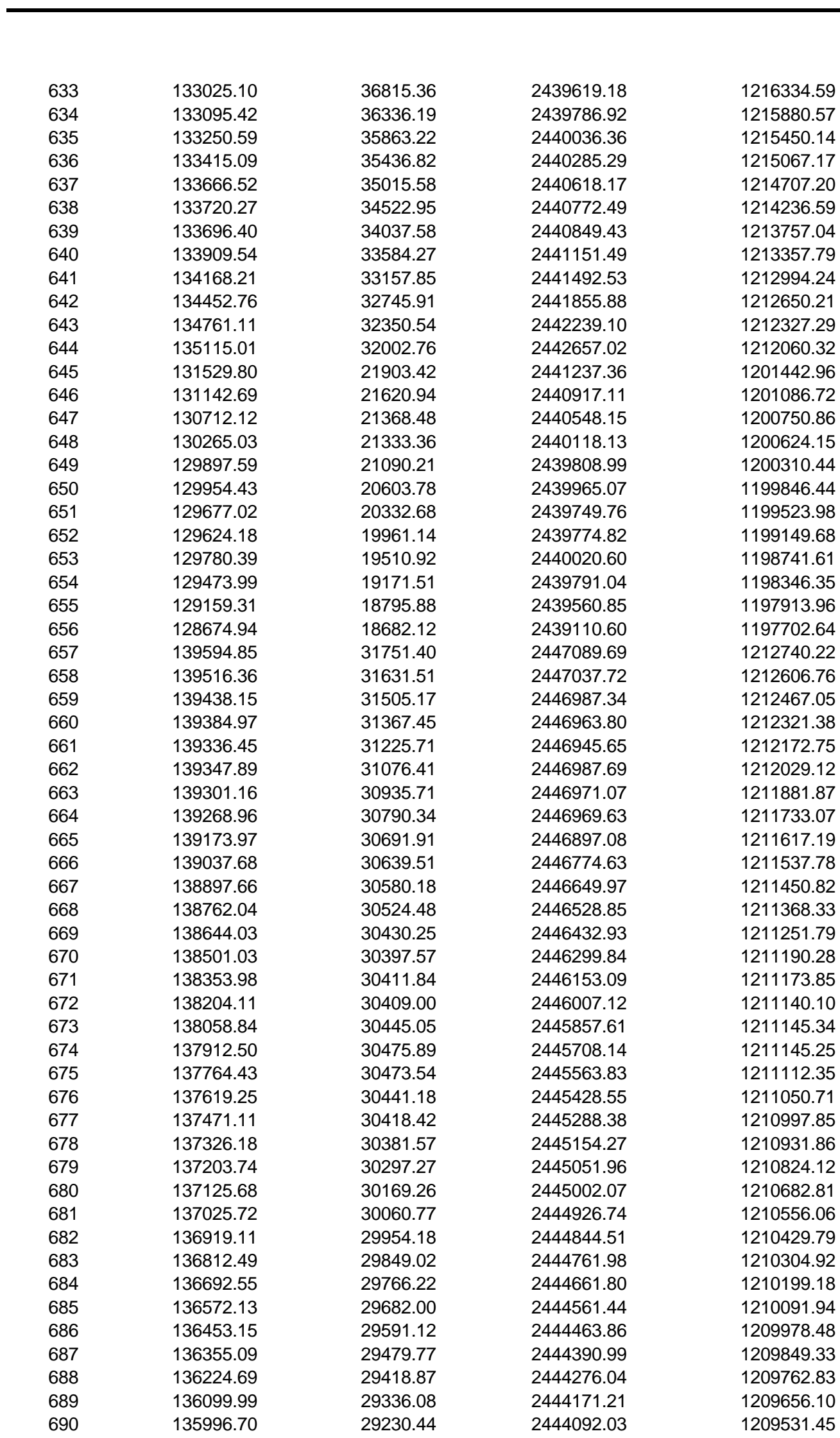


WSRC-TR-2002-00470

October 2002

\begin{tabular}{|c|c|c|c|c|}
\hline 691 & 135893.42 & 29120.05 & 2444013.83 & 1209402.15 \\
\hline 692 & 135781.09 & 29021.07 & 2443924.44 & 1209282.15 \\
\hline 693 & 135713.49 & 28891.16 & 2443885.18 & 1209141.14 \\
\hline 694 & 135624.96 & 28770.77 & 2443823.47 & 1209005.11 \\
\hline 695 & 135527.86 & 28657.51 & 2443751.92 & 1208874.30 \\
\hline 696 & 135444.56 & 28531.41 & 2443696.51 & 1208733.77 \\
\hline 697 & 135373.15 & 28399.59 & 2443653.92 & 1208590.11 \\
\hline 698 & 135291.76 & 28275.39 & 2443599.98 & 1208451.83 \\
\hline 699 & 135254.14 & 28131.67 & 2443592.89 & 1208303.51 \\
\hline 700 & 135190.83 & 27995.10 & 2443559.19 & 1208156.86 \\
\hline 701 & 135148.88 & 27856.82 & 2443546.74 & 1208012.97 \\
\hline 702 & 135149.81 & 27706.91 & 2443578.62 & 1207866.56 \\
\hline 703 & 135141.70 & 27557.95 & 2443601.47 & 1207719.21 \\
\hline 704 & 135105.03 & 27414.23 & 2443595.31 & 1207571.09 \\
\hline 705 & 135099.78 & 27265.27 & 2443620.95 & 1207424.33 \\
\hline 706 & 135105.94 & 27114.89 & 2443658.05 & 1207278.53 \\
\hline 707 & 135066.90 & 26973.07 & 2443649.17 & 1207131.78 \\
\hline 708 & 134989.31 & 26846.01 & 2443599.54 & 1206991.49 \\
\hline 709 & 134968.35 & 26699.91 & 2443609.23 & 1206844.28 \\
\hline 710 & 134970.23 & 26552.38 & 2443641.56 & 1206700.39 \\
\hline 711 & 134958.31 & 26403.42 & 2443660.68 & 1206552.26 \\
\hline 712 & 134966.38 & 26253.99 & 2443699.45 & 1206407.78 \\
\hline 713 & 134998.72 & 26109.30 & 2443760.97 & 1206272.97 \\
\hline 714 & 134986.81 & 25961.30 & 2443779.90 & 1206125.77 \\
\hline 715 & 134975.84 & 25813.29 & 2443799.75 & 1205978.76 \\
\hline 716 & 134879.69 & 25703.85 & 2443728.34 & 1205851.86 \\
\hline 717 & 134866.34 & 25556.79 & 2443745.67 & 1205705.28 \\
\hline 718 & 134767.81 & 25444.02 & 2443672.61 & 1205574.63 \\
\hline 719 & 134660.72 & 25342.66 & 2443588.82 & 1205453.39 \\
\hline 720 & 134560.76 & 25232.74 & 2443513.78 & 1205325.23 \\
\hline 721 & 134536.47 & 25084.74 & 2443520.60 & 1205175.47 \\
\hline 722 & 134478.42 & 24955.33 & 2443490.57 & 1205036.91 \\
\hline 723 & 134358.96 & 24868.25 & 2443391.72 & 1204927.07 \\
\hline 724 & 134262.33 & 24758.81 & 2443319.84 & 1204800.08 \\
\hline 725 & 134187.12 & 24628.42 & 2443273.22 & 1204657.02 \\
\hline 726 & 134062.89 & 24545.15 & 2443168.94 & 1204549.92 \\
\hline 727 & 133932.96 & 24471.88 & 2443057.00 & 1204451.41 \\
\hline 728 & 133809.69 & 24388.14 & 2442953.75 & 1204344.05 \\
\hline 729 & 133694.98 & 24291.07 & 2442861.62 & 1204225.41 \\
\hline 730 & 133604.06 & 24180.19 & 2442795.61 & 1204098.20 \\
\hline 731 & 133536.46 & 24047.42 & 2442756.93 & 1203954.38 \\
\hline 732 & 133504.08 & 23901.80 & 2442755.35 & 1203805.27 \\
\hline 733 & 133465.04 & 23758.56 & 2442746.76 & 1203657.12 \\
\hline 734 & 133386.97 & 23640.54 & 2442694.79 & 1203525.57 \\
\hline 735 & 133366.48 & 23496.34 & 2442704.55 & 1203380.31 \\
\hline 736 & 133366.46 & 23345.96 & 2442735.60 & 1203233.23 \\
\hline 737 & 133362.16 & 23194.62 & 2442762.66 & 1203084.33 \\
\hline 738 & 133367.37 & 23048.99 & 2442797.85 & 1202942.98 \\
\hline 739 & 133368.78 & 22904.31 & 2442829.11 & 1202801.78 \\
\hline 740 & 133313.55 & 22765.35 & 2442803.81 & 1202654.47 \\
\hline 741 & 133279.74 & 22623.06 & 2442800.14 & 1202508.32 \\
\hline 742 & 133250.21 & 22478.39 & 2442801.16 & 1202360.73 \\
\hline 743 & 133215.45 & 22336.57 & 2442796.46 & 1202214.84 \\
\hline 744 & 133186.40 & 22199.99 & 2442796.26 & 1202075.26 \\
\hline 745 & 133128.32 & 22061.98 & 2442767.97 & 1201928.29 \\
\hline 746 & 133106.88 & 21926.83 & 2442774.93 & 1201791.68 \\
\hline 747 & 133032.62 & 21817.38 & 2442724.92 & 1201669.29 \\
\hline 748 & 132986.44 & 21682.23 & 2442707.67 & 1201527.57 \\
\hline
\end{tabular}




\begin{tabular}{|c|c|c|c|c|}
\hline 749 & 132887.91 & 21570.88 & 2442634.31 & 1201398.31 \\
\hline 750 & 132753.69 & 21525.69 & 2442512.39 & 1201326.38 \\
\hline 751 & 132634.22 & 21437.18 & 2442413.83 & 1201215.14 \\
\hline 752 & 132542.83 & 21328.21 & 2442346.96 & 1201089.69 \\
\hline 753 & 132430.03 & 21231.15 & 2442256.69 & 1200971.44 \\
\hline 754 & 132299.14 & 21165.49 & 2442142.24 & 1200880.19 \\
\hline 755 & 132228.69 & 21037.48 & 2442099.78 & 1200740.44 \\
\hline 756 & 132162.99 & 20908.04 & 2442062.27 & 1200600.27 \\
\hline 757 & 132082.07 & 20811.92 & 2442002.99 & 1200489.54 \\
\hline 758 & 132062.06 & 20670.10 & 2442012.72 & 1200346.70 \\
\hline 759 & 132064.89 & 20522.09 & 2442046.06 & 1200202.53 \\
\hline 760 & 131990.63 & 20397.89 & 2441999.09 & 1200065.71 \\
\hline 761 & 131869.74 & 20341.27 & 2441892.55 & 1199985.36 \\
\hline 762 & 131771.21 & 20236.11 & 2441817.91 & 1199862.16 \\
\hline 763 & 131743.11 & 20096.20 & 2441819.33 & 1199719.51 \\
\hline 764 & 131701.68 & 19954.38 & 2441808.12 & 1199572.25 \\
\hline 765 & 131637.89 & 19821.61 & 2441773.15 & 1199429.21 \\
\hline 766 & 131537.45 & 19709.79 & 2441698.02 & 1199299.09 \\
\hline 767 & 131411.32 & 19627.48 & 2441591.67 & 1199192.53 \\
\hline 768 & 131277.58 & 19563.73 & 2441474.03 & 1199102.55 \\
\hline 769 & 131156.21 & 19475.22 & 2441373.61 & 1198990.92 \\
\hline 770 & 131038.65 & 19382.44 & 2441277.79 & 1198875.88 \\
\hline 771 & 130941.54 & 19268.71 & 2441206.31 & 1198744.59 \\
\hline 772 & 130828.73 & 19170.69 & 2441116.23 & 1198625.41 \\
\hline 773 & 130683.57 & 19135.50 & 2440981.53 & 1198561.00 \\
\hline 774 & 130532.71 & 19123.62 & 2440836.42 & 1198518.22 \\
\hline 775 & 130390.40 & 19079.39 & 2440706.38 & 1198445.56 \\
\hline 776 & 130267.13 & 18993.27 & 2440603.60 & 1198335.86 \\
\hline 777 & 130147.66 & 18900.96 & 2440505.82 & 1198220.90 \\
\hline 778 & 130020.10 & 18820.55 & 2440397.67 & 1198115.90 \\
\hline 779 & 129890.64 & 18744.43 & 2440286.77 & 1198014.70 \\
\hline 780 & 129780.21 & 18645.45 & 2440199.21 & 1197895.09 \\
\hline 781 & 129679.30 & 18536.96 & 2440122.93 & 1197768.13 \\
\hline 782 & 129552.22 & 18460.36 & 2440014.45 & 1197666.96 \\
\hline 783 & 129426.09 & 18381.38 & 2439907.41 & 1197563.65 \\
\hline 784 & 129354.68 & 18250.51 & 2439864.60 & 1197420.91 \\
\hline 785 & 129322.77 & 18102.04 & 2439864.06 & 1197269.09 \\
\hline 786 & 129370.82 & 17966.87 & 2439938.97 & 1197146.81 \\
\hline 787 & 129466.93 & 17852.63 & 2440056.58 & 1197054.94 \\
\hline 788 & 129567.33 & 17740.78 & 2440177.88 & 1196966.27 \\
\hline 789 & 129667.73 & 17630.35 & 2440298.89 & 1196879.00 \\
\hline 790 & 129765.75 & 17518.97 & 2440417.77 & 1196790.31 \\
\hline 791 & 129852.35 & 17398.07 & 2440527.45 & 1196689.96 \\
\hline 792 & 129913.72 & 17259.57 & 2440616.08 & 1196567.17 \\
\hline 793 & 129905.60 & 17112.51 & 2440638.52 & 1196421.66 \\
\hline 794 & 129919.38 & 16969.74 & 2440681.50 & 1196284.85 \\
\hline 795 & 129983.13 & 16834.09 & 2440771.87 & 1196165.35 \\
\hline 796 & 130025.94 & 16711.77 & 2440839.01 & 1196054.56 \\
\hline 797 & 130036.39 & 16563.29 & 2440879.91 & 1195911.48 \\
\hline 798 & 137600.99 & 24338.39 & 2446671.84 & 1205078.82 \\
\hline 799 & 137208.87 & 24076.53 & 2446342.47 & 1204741.68 \\
\hline 800 & 136985.38 & 23645.18 & 2446213.04 & 1204273.65 \\
\hline 801 & 136969.43 & 23180.65 & 2446293.44 & 1203816.05 \\
\hline 802 & 137040.99 & 22696.42 & 2446463.49 & 1203357.26 \\
\hline 803 & 137217.10 & 22232.71 & 2446731.56 & 1202940.15 \\
\hline 804 & 137541.46 & 21887.08 & 2447120.22 & 1202669.16 \\
\hline 805 & 137701.48 & 21418.66 & 2447373.52 & 1202244.11 \\
\hline 806 & 137846.26 & 20974.80 & 2447606.85 & 1201839.93 \\
\hline
\end{tabular}


WSRC-TR-2002-00470

October 2002

\begin{tabular}{|c|c|c|c|c|}
\hline 807 & 137767.42 & 20498.79 & 2447628.12 & 1201358.09 \\
\hline 808 & 137537.81 & 20067.69 & 2447492.66 & 1200889.01 \\
\hline 809 & 137409.00 & 19595.92 & 2447464.18 & 1200400.98 \\
\hline 810 & 137271.71 & 19119.92 & 2447428.28 & 1199907.06 \\
\hline 811 & 137123.42 & 18672.72 & 2447375.67 & 1199439.03 \\
\hline 812 & 136837.08 & 18323.79 & 2447167.72 & 1199038.58 \\
\hline 813 & 136360.20 & 18256.09 & 2446715.30 & 1198873.82 \\
\hline 814 & 136011.19 & 18007.97 & 2446425.22 & 1198559.01 \\
\hline 815 & 135733.36 & 17607.46 & 2446236.26 & 1198109.88 \\
\hline 816 & 135455.48 & 17219.57 & 2446044.63 & 1197673.06 \\
\hline 817 & 135340.21 & 16744.41 & 2446030.08 & 1197184.50 \\
\hline 818 & 135299.48 & 16270.09 & 2446088.27 & 1196712.16 \\
\hline 819 & 134992.80 & 15907.61 & 2445863.21 & 1196294.25 \\
\hline 820 & 134736.94 & 15489.22 & 2445699.41 & 1195832.15 \\
\hline 821 & 134668.26 & 15121.79 & 2445708.17 & 1195458.58 \\
\hline 822 & 135093.83 & 15020.86 & 2446145.28 & 1195447.80 \\
\hline 823 & 135560.33 & 15079.20 & 2446589.50 & 1195601.27 \\
\hline 824 & 136017.85 & 15160.93 & 2447020.12 & 1195775.76 \\
\hline 825 & 136474.26 & 15053.16 & 2447488.80 & 1195764.68 \\
\hline 826 & 136932.71 & 14988.23 & 2447950.63 & 1195795.92 \\
\hline 827 & 137326.99 & 14740.58 & 2448387.46 & 1195635.18 \\
\hline 828 & 137668.12 & 14451.47 & 2448780.87 & 1195422.91 \\
\hline 829 & 138038.45 & 14158.96 & 2449203.55 & 1195213.35 \\
\hline 830 & 138394.20 & 13869.45 & 2449611.35 & 1195003.71 \\
\hline 831 & 138789.28 & 13613.00 & 2450050.79 & 1194834.54 \\
\hline 832 & 139221.46 & 13415.98 & 2450514.24 & 1194731.17 \\
\hline 833 & 139641.15 & 13193.55 & 2450970.72 & 1194600.37 \\
\hline 834 & 140055.36 & 12984.23 & 2451419.14 & 1194481.25 \\
\hline 835 & 140469.40 & 12764.88 & 2451869.46 & 1194352.30 \\
\hline 836 & 140643.70 & 12372.90 & 2452120.98 & 1194004.93 \\
\hline 837 & 140852.59 & 12028.39 & 2452396.52 & 1193711.14 \\
\hline 838 & 141160.02 & 11834.49 & 2452737.32 & 1193585.03 \\
\hline 839 & 141520.83 & 11660.79 & 2453126.15 & 1193489.73 \\
\hline 840 & 141070.39 & 17765.63 & 2451423.49 & 1199367.68 \\
\hline 841 & 141229.57 & 17300.59 & 2451675.31 & 1198945.75 \\
\hline 842 & 141351.48 & 16869.43 & 2451883.68 & 1198549.26 \\
\hline 843 & 141342.94 & 16379.01 & 2451976.71 & 1198067.83 \\
\hline 844 & 141358.97 & 15909.75 & 2452089.40 & 1197612.17 \\
\hline 845 & 141500.36 & 15447.25 & 2452323.30 & 1197189.05 \\
\hline 846 & 141589.23 & 15008.48 & 2452500.94 & 1196778.26 \\
\hline 847 & 141728.08 & 14534.97 & 2452734.64 & 1196343.83 \\
\hline 848 & 141589.10 & 14091.99 & 2452690.29 & 1195881.83 \\
\hline 849 & 141334.93 & 13669.35 & 2452529.06 & 1195415.90 \\
\hline 850 & 141212.05 & 13188.26 & 2452508.32 & 1194919.93 \\
\hline 851 & 141128.97 & 12710.54 & 2452525.83 & 1194435.50 \\
\hline 852 & 141128.89 & 12219.26 & 2452627.32 & 1193954.95 \\
\hline 853 & 141351.60 & 11792.32 & 2452933.43 & 1193583.40 \\
\hline 854 & 141726.81 & 11563.56 & 2453347.72 & 1193437.22 \\
\hline 855 & 142152.85 & 11418.66 & 2453794.40 & 1193383.57 \\
\hline 856 & 142225.63 & 11009.53 & 2453950.18 & 1192998.43 \\
\hline 857 & 151865.64 & 26059.07 & 2460266.08 & 1209710.76 \\
\hline 858 & 151992.64 & 25594.04 & 2460486.47 & 1209282.27 \\
\hline 859 & 152235.70 & 25188.30 & 2460808.09 & 1208935.76 \\
\hline 860 & 152492.31 & 24857.93 & 2461127.37 & 1208665.77 \\
\hline 861 & 152815.84 & 24536.03 & 2461510.35 & 1208417.89 \\
\hline 862 & 153197.83 & 24347.95 & 2461922.81 & 1208312.99 \\
\hline 863 & 153557.81 & 24229.33 & 2462299.38 & 1208271.45 \\
\hline 864 & 153835.61 & 24031.09 & 2462612.06 & 1208135.06 \\
\hline
\end{tabular}




\begin{tabular}{|c|c|c|c|c|}
\hline 865 & 153922.82 & 23706.67 & 2462764.46 & 1207835.84 \\
\hline 866 & 153965.13 & 23267.91 & 2462896.61 & 1207415.51 \\
\hline 867 & 154131.11 & 22860.47 & 2463143.21 & 1207051.39 \\
\hline 868 & 154399.57 & 22465.73 & 2463487.42 & 1206720.90 \\
\hline 869 & 154568.09 & 22034.57 & 2463741.42 & 1206334.11 \\
\hline 870 & 154736.60 & 21581.39 & 2463999.98 & 1205925.78 \\
\hline 871 & 154822.11 & 21162.95 & 2464170.18 & 1205534.25 \\
\hline 872 & 154829.69 & 20755.52 & 2464261.88 & 1205137.37 \\
\hline 873 & 154915.20 & 20353.17 & 2464428.75 & 1204761.57 \\
\hline 874 & 155066.79 & 20069.40 & 2464635.72 & 1204515.41 \\
\hline 875 & 155154.00 & 19686.53 & 2464800.22 & 1204159.02 \\
\hline 876 & 155405.53 & 19355.31 & 2465114.75 & 1203887.14 \\
\hline 877 & 155559.66 & 19013.94 & 2465336.11 & 1203585.17 \\
\hline 878 & 155836.62 & 18783.52 & 2465654.65 & 1203417.12 \\
\hline 879 & 156200.83 & 18644.57 & 2466039.60 & 1203356.59 \\
\hline 880 & 156274.48 & 18250.69 & 2466193.14 & 1202986.61 \\
\hline 881 & 156473.50 & 17883.90 & 2466463.67 & 1202669.07 \\
\hline 882 & 156615.76 & 17471.37 & 2466688.17 & 1202295.06 \\
\hline 883 & 156947.78 & 17219.77 & 2467064.95 & 1202117.69 \\
\hline 884 & 157145.95 & 16863.99 & 2467332.38 & 1201810.74 \\
\hline 885 & 157220.45 & 16476.88 & 2467485.35 & 1201447.56 \\
\hline 886 & 157348.32 & 16109.25 & 2467686.49 & 1201114.47 \\
\hline 887 & 157416.04 & 15743.32 & 2467828.45 & 1200770.60 \\
\hline 888 & 157464.29 & 15418.05 & 2467942.95 & 1200462.46 \\
\hline 889 & 157593.85 & 15058.89 & 2468143.99 & 1200137.99 \\
\hline 890 & 157871.66 & 14846.25 & 2468459.71 & 1199987.52 \\
\hline 891 & 157928.38 & 14482.01 & 2468590.56 & 1199643.02 \\
\hline 892 & 158024.90 & 14067.79 & 2468770.68 & 1199257.87 \\
\hline 893 & 158021.47 & 13699.33 & 2468843.59 & 1198896.78 \\
\hline 894 & 158063.78 & 13291.89 & 2468969.29 & 1198507.04 \\
\hline 895 & 158214.52 & 12881.90 & 2469201.57 & 1198137.24 \\
\hline 896 & 158254.30 & 12563.41 & 2469306.39 & 1197833.96 \\
\hline 897 & 158344.05 & 12269.47 & 2469455.01 & 1197565.05 \\
\hline 898 & 158155.13 & 11952.69 & 2469335.79 & 1197216.12 \\
\hline 899 & 158257.58 & 11595.22 & 2469509.99 & 1196887.69 \\
\hline 900 & 158261.79 & 11287.74 & 2469577.73 & 1196587.82 \\
\hline 901 & 158333.75 & 10941.29 & 2469719.82 & 1196263.85 \\
\hline 902 & 158293.90 & 10594.00 & 2469752.73 & 1195915.92 \\
\hline 903 & 158168.50 & 10204.36 & 2469710.71 & 1195508.87 \\
\hline 904 & 158193.03 & 9876.55 & 2469802.55 & 1195193.31 \\
\hline 905 & 158066.77 & 9475.05 & 2469762.16 & 1194774.47 \\
\hline 906 & 157733.00 & 9206.57 & 2469491.26 & 1194442.79 \\
\hline 907 & 157384.83 & 8888.11 & 2469216.61 & 1194059.24 \\
\hline 908 & 157009.55 & 8622.18 & 2468904.58 & 1193721.46 \\
\hline 909 & 156733.37 & 8330.82 & 2468694.75 & 1193379.32 \\
\hline 910 & 156356.42 & 8195.33 & 2468354.07 & 1193168.78 \\
\hline 911 & 156036.20 & 7964.12 & 2468088.71 & 1192876.36 \\
\hline 912 & 155813.45 & 8173.37 & 2467827.52 & 1193034.93 \\
\hline 913 & 155405.17 & 8195.44 & 2467423.60 & 1192972.03 \\
\hline 914 & 155119.67 & 7905.78 & 2467204.29 & 1192629.62 \\
\hline 915 & 154691.88 & 7672.04 & 2466834.22 & 1192312.47 \\
\hline 916 & 154375.91 & 7530.62 & 2466554.42 & 1192108.76 \\
\hline 917 & 154066.70 & 7301.10 & 2466299.46 & 1191820.27 \\
\hline 918 & 153728.70 & 7076.67 & 2466015.28 & 1191530.81 \\
\hline 919 & 153427.10 & 6701.46 & 2465797.91 & 1191101.39 \\
\hline 920 & 153115.34 & 6334.72 & 2465568.84 & 1190678.15 \\
\hline 921 & 152810.36 & 6020.49 & 2465335.53 & 1190307.68 \\
\hline 922 & 152872.99 & 5608.81 & 2465481.98 & 1189917.95 \\
\hline
\end{tabular}


WSRC-TR-2002-00470

October 2002

$\begin{array}{lllll}923 & 152672.18 & 5203.94 & 2465369.33 & 1189480.36 \\ 924 & 152537.45 & 4781.27 & 2465324.99 & 1189039.04 \\ 925 & 152367.99 & 4480.59 & 2465221.45 & 1188709.85 \\ 926 & 152144.33 & 4174.82 & 2465065.92 & 1188364.48 \\ 927 & 152118.86 & 3687.76 & 2465141.78 & 1187882.76 \\ 928 & 152018.85 & 3316.76 & 2465120.72 & 1187499.16\end{array}$

